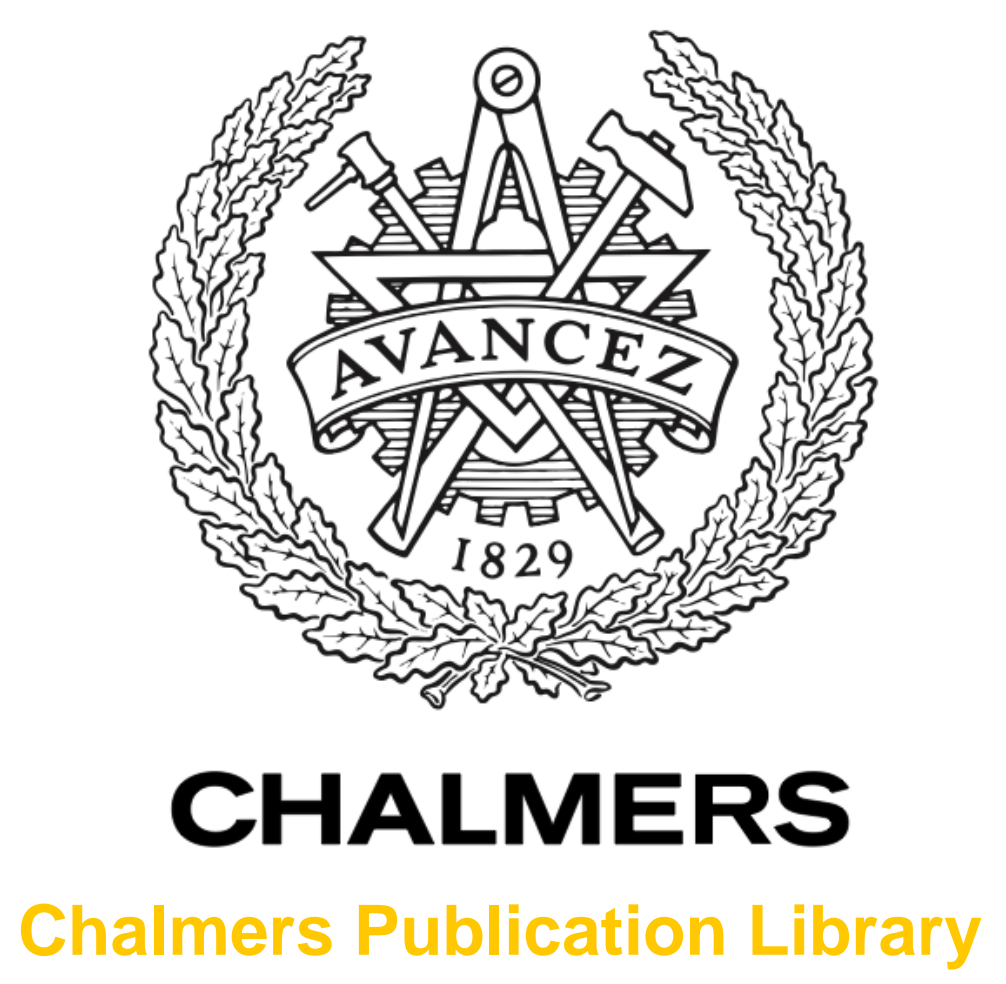

\title{
On the Average Rate of HARQ-Based Quasi-Static Spectrum Sharing Networks
}

This document has been downloaded from Chalmers Publication Library (CPL). It is the author's version of a work that was accepted for publication in:

IEEE Transactions on Wireless Communications (ISSN: 1536-1276)

Citation for the published paper:

Makki, B. ; Eriksson, T. (2012) "On the Average Rate of HARQ-Based Quasi-Static

Spectrum Sharing Networks". IEEE Transactions on Wireless Communications, vol. 11(1), pp. 65-77.

http://dx.doi.org/10.1109/TWC.2011.110811.10170

0

Down「oaded from: http://publications.lib.chalmers.se/publication/155368

Notice: Changes introduced as a result of publishing processes such as copy-editing and formatting may not be reflected in this document. For a definitive version of this work, please refer to the published source. Please note that access to the published version might require a subscription. 


\title{
On the average rate of HARQ-based quasi-static spectrum sharing networks
}

\author{
Behrooz Makki and Thomas Eriksson \\ Email: behrooz.makki@chalmers.se,thomase@chalmers.se
}

\begin{abstract}
Spectrum sharing networks are communication setups in which unlicensed secondary users are permitted to work within the spectrum resources of primary licensees. Considering quasi-static fading environments, this paper studies the effect of hybrid automatic repeat request (HARQ) feedback on the average rate of unlicensed spectrum sharing channels. The results are obtained for different scenarios; Under both peak and average secondary user transmission power constraints, the channel average rate is determined under primary user limited received interference power conditions when there is perfect information about the interference available at the secondary user transmitter. An approximate solution for power allocation between incremental redundancy (INR) HARQ-based data retransmissions is proposed which can be applied in single-user networks as well. Then, we investigate the effect of imperfect secondary-primary channel state information on the interferencelimited average rate of the secondary channel. Finally, we restudy all mentioned scenarios in the case where the data transmission is constrained to have limited outage probability. Substantial performance improvement is observed with even a single HARQbased retransmission in all simulations.
\end{abstract}

\section{INTRODUCTION}

Spectrum is a scarce valuable resource in today wireless communication networks; with ever-increasing number of wireless devices communicating at high data rates, there is growing demand for spectrum resources. This point has led to complaints about spectrum shortage which is expected to grow even more in the coming years. On the other hand, recent studies have shown that the shortage comes mainly from outdated resource allocation policies where, at any given instant and location, large portions of spectrum are underutilized by licensed users that allow little sharing [1], [2]. This is the main motivation for the spectrum sharing concept that has attracted plenty of studies in the last decade.

In general, the goal of spectrum sharing methods, normally modeled as interference [3]-[5], cooperative [6], [7] or cognitive radio [8]-[12] networks, is to better utilize the radio spectrum by allowing the unlicensed secondary users (SU's) to coexist with the licensed primary users (PU's). Along with the standard interference channel, in which two independent transmitters transmit independent messages to two independent receivers, there are other ways to exploit the idea of spectrum sharing. For instance, in a method normally called as interference-avoiding paradigm [12]-[14], provided that the secondary transmitter can sense the spatial, temporal or spectral gaps of the primary resources, it can adjust its transmission parameters to fill these white spaces. Although this approach can theoretically lead to significant spectral efficiency improvement, it suffers from some practical shortcomings mainly related to imperfect gap detection. In another way, widely referred to as simultaneous or controlled transmission [15], [16], a secondary user can simultaneously coexist with a primary user as long as it works under a certain interference level imposed by the primary user quality-ofservice requirements. In these methods, limits on the interference level received at the PU receiver, which is normally called as interference temperature, can be considered to be long-term average or short-term peak constraints. Finally, there are the cooperative networks in which the two users normally know each others messages prior to transmission, and can potentially help each other to improve the overall efficiency.

Reviewing the literature, a large number of scientific reports can be found that have tackled the spectrum sharing, particularly the cognitive radio, problem in different theoretical aspects. In [11] and [12], the authors presented some information theoretic models, limits and open problems of spectrum sharing networks. Assuming perfect SU-PU channel state information (CSI), [17] investigated the effect of optimal power allocation on the capacity of secondary channel under different power constraints. With both peak and average interference power constraints, [18] studied the capacity of the secondary channel in the case where all channels are fully known by the SU transmitter. In [19], considering a path loss shadowfading model with multiple primary and secondary users, the system-level capacities of spectrum sharing networks under an average interference power constraint were studied.

Removing the assumptions, recent works are moving toward more realistic system models; allowing limited interference power at the PU receiver, Ji et al., [20] analyzed the capacity of multicast spectrum sharing networks. In their work, while the SU-SU link is assumed to be perfectly known, the results are obtained for the cases where the interference information is perfectly or imperfectly available at the SU transmitter. Also, [21] studied the ergodic, the outage, and the minimumrate capacity of spectrum sharing network under average and peak interference constraints. This work which was based on perfect SU-PU channel state information was later extended by [22] in which the same results were obtained under imperfect CSI feedback assumption. Considering different channel information imperfection model and interference constraints, [23] presented the same results as [22] and verified the effect of feedback quantization as well. Finally, among other works dealing with imperfect SU-PU channel state information we can mention [24] in which the secondary channel outage capacity lower bounds were obtained under the constraint 
that the PU is provided with a minimum rate for a certain percentage of time.

References [17]-[24] are all based on the assumption that the secondary user has perfect knowledge of the link between its transmitter and receiver. Channel state estimation at the receiver is relatively simple and incurs negligible loss in the transmission rate, particularly when the channels experience slow variations. However, due to the signaling load caused by reporting the channel information, assuming perfect knowledge at, e.g., secondary, transmitter is an optimistic assumption, which does not match with reality. This is the motivation for the present limited channel quality information feedback systems [25], [26], and for this paper as well.

Generally, there are two different approaches, namely, quantized CSI feedback [27]-[30] and automatic repeat request (ARQ) [30]-[36], providing the imperfect channel quality information at the transmitter. Quantized CSI feedback belongs to the physical layer techniques and, in order to exploit its properties, needs some additional designs, particularly at the transmitter [37]. This point increases the system complexity and cost. ARQ, on the other hand, is a technique in the data link layer which is already provided in most of wireless protocols. Therefore, it needs no additional closedloop design which introduces it as a cost- and complexityefficient approach. In a general ARQ approach, the transmitter considers some initial transmission rate and power with no pre-knowledge about the channel quality. Then, with the help of ARQ, the decoding status at the receiver will be reported back to the transmitter via one bit feedback. The feedback indicates successful decoding of the received signal by an acknowledgement (ACK) bit if $R_{T} \leq R$ and failed decoding by negative acknowledgement (NACK) if $R_{T}>R$ where $R_{T}$ and $R$ are the transmission and maximum possible rates, respectively. Based on the received feedback, it is decided by the transmitter whether to retransmit the data or to move on to the next codeword. In basic ARQ schemes the same data is retransmitted and the receiver decodes the message based on the received signal in each time slot. Hybrid ARQ (HARQ) protocols, on the other hand, are more advanced methods where, while utilizing both forward error correction and error detection, the receiver combines all received representations of a message. Finally, there are a number of HARQ protocols such as Repetition Time Diversity (RTD) and INcremental Redundancy (INR) [31]-[33] mainly differing in the retransmission and message decoding procedures.

While there are many scientific reports dealing with single user ARQ-based networks, recent studies show new trends on implementation of ARQ feedback in spectrum sharing systems. For instance, [38] used the PU ARQ feedback delivered at the secondary transmitter to improve the PU protection. Ao and Chen [39] developed a novel HARQ scheme for endto-end error control in cognitive radio networks. Moreover, [40] studied the effect of primary link ARQ feedback on the performance of spectrum sharing networks. There, the PU ARQ feedback is given not only to the primary transmitter, which exploits it for retransmission decision-making, but also to the SU transmitter and receiver. Having information about the PU receiver decoding status lets the SU to adjust its rate appropriately. Then, with slightly different conditions, [41] investigated the effect of PU ARQ feedback on the data transmission efficiency of a secondary user utilizing a clear-to-send feedback scheme. Also, Narasimhan [6] obtained the outage probability and a sum-throughput upper bound of interference channels having cooperation at the receivers. In his work, both users are equipped with ARQ feedback and the user who decodes its message first works as a relay for the other one. Among other ARQ-based cooperative network approaches one can mention [7] and [42] in which different cooperative ARQ protocols were evaluated.

In this perspective, this paper aims to study the effect of HARQ feedback on the average rate of unlicensed quasi-static spectrum sharing channels. The main constraint is to keep the PU received interference power below a given threshold. However, in Section V the outage probability is also considered as an additional quality-of-service requirement. To be more specific, the results are obtained under different scenarios listed as follows:

- Section III: (Average rate with limited PU received interference power and perfect SU-PU CSI feedback) This section focuses on the following optimization problem

$$
\begin{aligned}
& \max \bar{R}^{\mathrm{s}} \\
& \text { subject to } \bar{P}^{\mathrm{s}} \leq P
\end{aligned}
$$

in which $\bar{R}^{\mathrm{s}}$ is the $\mathrm{SU}$ average transmission rate and $\bar{P}^{\mathrm{s}}$ denotes its average transmission power constrained to be less than $P$. There are different situations in which this problem is of interest; Assuming perfect SUPU CSI (or nonfading AWGN SU-PU channel model), the PU received interference power is directly related to the SU transmission power and can be considered instead. Further, the problem setup represents the longterm performance of a user in a standard interference network. The same setup also represents the cases where the PU received interference constraint is so relaxed or the cognitive radio transmission power is so small that the presence of cognitive radio is tolerable by the licensed user. Finally, as discussed in Section V, some other PU quality-of-service requirements, e.g., the PU outage probability constraint, can be easily mapped to this problem.

Here, considering long-term constraints, an approximate solution for power allocation between INR HARQ-based data retransmissions is proposed which can be also applied in single-user networks utilizing variable-lengthcodeword HARQ feedback [30], [31]. Finally, we study the channel average rate under no and infinitely many HARQ feedback bits which demonstrates the channel performance with no SU-SU CSI and in delay-insensitive systems, respectively.

- Section IV: (Average rate with limited PU received interference power and imperfect SU-PU CSI feedback) The perfect SU-PU CSI assumption is removed in this part where the channel performance is studied under the PU limited received interference power conditions. Again, the results are obtained under different no and infinitely many 
ARQ feedback cases and we study the effect of the PU tolerability on the achievable rates of the SU-SU channel.

- Section V: (Average rate in outage probability limited conditions) Here, we restudy all mentioned scenarios in the case where the data transmission is constrained to have limited outage probability. The outage probability constraint is considered for both the primary and the secondary users under different SU-PU CSI conditions.

Simulation results are mainly presented for Rayleigh-fading channels. The simulations indicate substantial performance improvement with even a single HARQ-based retransmission.

An efficient way to read this paper is to first read Section II, "System model". As a second step, read Section VI where the most important results of the paper are summarized, with references to the respective figures. Finally, the mathematical parts, Sections III-V, can be studied for a deeper understanding.

\section{SYSTEM MODEL}

As illustrated in Fig. 1, our channel model consists of two primary and secondary communication links which share the same narrow-band frequency with bandwidth $B$. For simplicity and with no loss of generality, we consider $B=1$. Let $g^{\mathrm{pp}}=\left|h^{\mathrm{pp}}\right|^{2}, g^{\mathrm{ps}}=\left|h^{\mathrm{ps}}\right|^{2}, g^{\mathrm{sp}}=\left|h^{\mathrm{sp}}\right|^{2}$ and $g^{\mathrm{ss}}=\left|h^{\mathrm{ss}}\right|^{2}$ be the instantaneous channel gains of the PU-PU, PU-SU, SU-PU and SU-SU links, respectively. While simulation results are mainly obtained for Rayleigh-fading channels, the analytical results are valid for any combination of independent positive random variables. We consider quasi-static conditions, where the channel gains remain constant for a duration of $t_{c}$, generally determined by the channels coherence time, and then change independently according to their corresponding fading probability density functions (pdfs) $f_{g^{\mathrm{pp}}}, f_{g^{\mathrm{ps}}}, f_{g^{\mathrm{sp}}}$ and $f_{g^{\mathrm{ss}}} . t_{c}$ is supposed to be much larger than the length of the codewords containing $K$ nats information ${ }^{1}$. In this way, multiple packets, each one having several ARQ rounds, are sent within one coherent period. When the channels are in good conditions, many packets can be sent within the coherent period, while only few can be transmitted within the same period for bad channel conditions. Moreover, we focus on backlogged transmitter systems where there is an infinite amount of information to be transmitted and so, the communication is continuous [43]. Consequently, we can consider the average rate as a valid performance yardstick [30], [31]. The AWGN at the primary and secondary receivers are denoted by $n^{\mathrm{p}}$ and $n^{\mathrm{s}}$, respectively, and have common distributions $\mathcal{C N}\left(0, \sigma^{2}\right)$ (circularly symmetric complex Gaussian variables with zero mean and variance $\sigma^{2}$ for bandwidth $B$ ). Finally, it is assumed that the primary transmitter is communicating at fixed power $P^{\mathrm{p}}$ and has Gaussian input distribution.

This is an appropriate model of stationary or slow-moving users such as wireless local area networks (WLANs) [44]. Particularly, since long-block-length capacity-approaching codes can be implemented in such systems, the results can provide realistic insight about the performance bounds of the considered channel quality feedback approaches.

\footnotetext{
${ }^{1}$ All results are presented in natural logarithm basis. Also, in all simulation results the average rate is presented in nats-per-channel-use (npcu).
}

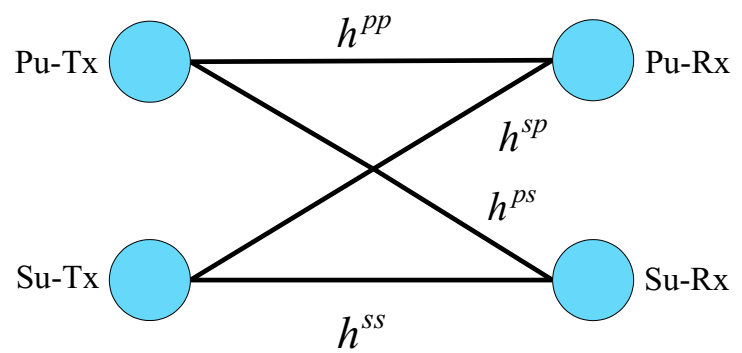

Figure 1. Channel model. The channels share the same narrow-band frequency with bandwidth $\mathrm{B}$.

\section{A. HARQ feedback scheme}

As the best ARQ feedback scheme, reaching the highest average rates [30], [31], we consider the INcremental Redundancy (INR) HARQ protocol where, at each retransmission request, the transmitter sends new redundancy bits and the receiver combines them. In this way, considering $M+1$ maximum allowable ARQ rounds, i.e., maximum $M$ bits ARQ feedback and retransmission rounds, $K$ information nats is encoded into a codeword of length $T_{(M+1)}$. Then, the codeword is serially punctured into $M+1$ sub-codewords with strictly decreasing rates ${ }^{2}$

$$
R_{m}^{\mathrm{s}}=\frac{K}{\sum_{k=1}^{m} T_{k}}, m=1, \ldots, M+1 .
$$

Here, $T_{m}$ and $R_{m}^{\mathrm{s}}$ are the secondary user channel uses and the equivalent transmission rate in the $m$-th time slot, respectively. Moreover, $T_{(m)}=\sum_{k=1}^{m} T_{k}$ denotes the total number of channel uses at the end of the $m$-th slot.

At the end of each (re)transmission, 1 bit ARQ feedback is sent back from the secondary receiver to the secondary transmitter which indicates the corresponding message decoding status. Since the data rate for ARQ feedback is very low, it is assumed that the feedback is received error-free and with negligible delay. The retransmissions continue a maximum of $M$ times, in practice determined by the system delay requirements, where finally the lowest rate $R_{M+1}^{\mathrm{s}}=\frac{K}{T_{(M+1)}}$ is tried with no ARQ feedback. Outage occurs if the channel instantaneous gain can not support $R_{M+1}^{\mathrm{s}}$. Let us define $S_{m}$ as the event that the secondary user transmitted data is correctly decoded by the secondary receiver at the end of the $m$-th slot. In this way, the average rate of the secondary channel can be calculated by

$$
\bar{R}^{\mathrm{s}}=\sum_{m=1}^{M+1} R_{m}^{\mathrm{s}} \operatorname{Pr}\left\{\bar{S}_{1}, \ldots, \bar{S}_{m-1}, S_{m}\right\}
$$

in which $\bar{S}_{m}$ is the complement of the event $S_{m}$ and $S_{0}$ is the empty set. Assuming that the primary transmitter is communicating with power $P^{\mathrm{p}}$, the secondary channel signalto-interference-and-noise-ratio (SINR) at the $m$-th slot would be

$$
\gamma_{m}^{\mathrm{s}}=\frac{P_{m}^{\mathrm{s}} g^{\mathrm{ss}}}{P^{\mathrm{p}} g^{\mathrm{ps}}+\sigma^{2}}
$$

\footnotetext{
${ }^{2}$ Since each transmission experiences an AWGN channel, all results are restricted to Gaussian input distributions.
} 
in which $P_{m}^{\mathrm{s}}$ is the power considered for the $m$-th (re)transmission of the secondary transmitter. Therefore, implementing random coding and typical set-based decoding, we can use the results of [45] where $\operatorname{Pr}\left\{S_{m}\right\}$ is simplified to the Time Division Multiple Access (TDMA)-type equation

$$
\operatorname{Pr}\left\{S_{m}\right\}=\operatorname{Pr}\left\{\sum_{k=1}^{m} \frac{T_{k}}{\sum_{j=1}^{m} T_{j}} \log \left(1+\frac{P_{k}^{\mathrm{s}} g^{\mathrm{ss}}}{P^{\mathrm{p}} g^{\mathrm{ps}}+\sigma^{2}}\right) \geq R_{m}^{\mathrm{s}}\right\} .
$$

Note that, based on (1), we have

$$
\frac{T_{k}}{\sum_{j=1}^{m} T_{j}}=R_{m}^{\mathrm{s}}\left(\frac{1}{R_{k}^{\mathrm{s}}}-\frac{1}{R_{k-1}^{\mathrm{s}}}\right), R_{0}^{\mathrm{s}} \doteq \infty
$$

and so $\operatorname{Pr}\left\{S_{m}\right\}$ is found as a function of $R_{k}^{\mathrm{s}}$,s.

In the following sections, we study how the quality of SUPU CSI affects the power allocation, and correspondingly the average rate, of the secondary user under interference-limited conditions. Finally, note that implementing such a protocol mainly requires:

- A good mother code that can be punctured into different sub-codeword rates.

- A single decoder handling all rates so that the coding performance of sub-codewords are capacity approaching. Among different models developed for this purpose, we can mention, e.g., [46]-[49].

\section{SECONDARY ChanNEl AVERAGE RATE}

\section{OPTIMIZATION; PERFECT SU-PU CSI FEEDBACK AND}

\section{LIMITED PU RECEIVED INTERFERENCE POWER}

With perfect SU-PU CSI, the optimal transmission powers $P_{k}^{\mathrm{s}}$ are determined based on the power constraint; in general, the SU actual consumed power is a random variable given by

$$
P^{\mathrm{s}}\left(g^{\mathrm{ss}}, g^{\mathrm{ps}}\right)= \begin{cases}P_{(m)}^{\mathrm{s}} & \text { if } \bar{S}_{1}, \ldots, \bar{S}_{m-1}, S_{m} \\ P_{(M+1)}^{\mathrm{s}} & \text { if } \bar{S}_{1}, \ldots, \bar{S}_{M-1}, \bar{S}_{M}\end{cases}
$$

in which $P_{(m)}^{\mathrm{s}} \doteq \frac{\sum_{k=1}^{m} P_{k}^{\mathrm{s}} T_{k}}{\sum_{k=1}^{m} T_{k}}$ is the power up to the end of the $m$-th slot. Therefore, the average transmission power of the secondary user is obtained by

$$
\begin{aligned}
\bar{P}^{\mathrm{s}} & =\sum_{m=1}^{M} P_{(m)}^{\mathrm{s}} \operatorname{Pr}\left\{\bar{S}_{1}, \ldots, \bar{S}_{m-1}, S_{m}\right\} \\
& +P_{(M+1)}^{\mathrm{s}} \operatorname{Pr}\left\{\bar{S}_{1}, \ldots, \bar{S}_{M-1}, \bar{S}_{M}\right\} .
\end{aligned}
$$

Finally, note that in the case of uniform power allocation, i.e., $P_{k}^{\mathrm{s}}=P, \forall k=1 \ldots M+1$, the randomness of $P^{\mathrm{s}}\left(g^{\mathrm{ss}}, g^{\mathrm{ps}}\right)$ disappears and we have $P_{(m)}^{\mathrm{s}}=P, m=1 \ldots M+1$. Considering (2), (7) and the secondary user transmission power constraint $\bar{P}^{\mathrm{s}} \leq P$, the secondary link average rate maximization problem can be formulated as

$$
\begin{array}{r}
\bar{R}^{\mathrm{s}, \max }=\max _{\left\{P_{m}^{\mathrm{s}}, R_{m}^{\mathrm{s}}, m=1 \ldots M+1\right\}} \sum_{m=1}^{M+1} R_{m}^{\mathrm{s}} \operatorname{Pr}\left\{\bar{S}_{1}, \ldots, \bar{S}_{m-1}, S_{m}\right\} \\
\text { subject to } \sum_{m=1}^{M+1} P_{(m)}^{\mathrm{s}} \operatorname{Pr}\left\{\bar{S}_{1}, \ldots, \bar{S}_{m-1}, S_{m}\right\} \\
+P_{(M+1)}^{\mathrm{s}} \operatorname{Pr}\left\{\bar{S}_{1}, \ldots, \bar{S}_{M-1}, \bar{S}_{M}\right\} \leq P
\end{array}
$$

which is a complex optimization problem even in its simplest cases. In the following, we solve (8) under two different interpretations of the power constraint.

\section{A. Short-term power constraint}

Following, e.g., [29]-[31], the short-term power constraint implies $P_{m}^{\mathrm{s}}=P, \forall m=1 \ldots M+1$. Therefore, based on (1)(4), the achievable rate of the secondary channel is a random variable given by

$R^{\mathrm{s}}\left(g^{\mathrm{ss}}, g^{\mathrm{ps}}\right)=\left\{\begin{array}{ccc}R_{m}^{\mathrm{s}} & \text { if } R_{m}^{\mathrm{s}} \leq \log \left(1+\frac{P g^{\mathrm{ss}}}{P^{\mathrm{p}} g^{\mathrm{ps}}+\sigma^{2}}\right) \\ 0 & \text { otherwise } & <R_{m-1}^{\mathrm{s}}, m=1 \ldots M+1\end{array}\right.$

where $R_{0}^{\mathrm{s}} \doteq \infty$. Consequently, the secondary link average rate is obtained by

$$
\bar{R}^{\mathrm{s}}=\sum_{m=1}^{M+1} R_{m}^{\mathrm{s}} \operatorname{Pr}\left\{R_{m}^{\mathrm{s}} \leq \log \left(1+\frac{P g^{\mathrm{ss}}}{P^{\mathrm{p}} g^{\mathrm{ps}}+\sigma^{2}}\right)<R_{m-1}^{\mathrm{s}}\right\}
$$

which, defining $\vartheta_{m} \doteq e^{R_{m}^{\mathrm{s}}}-1$, can be rewritten as

$$
\begin{aligned}
\bar{R}^{\mathrm{s}} & =\sum_{m=1}^{M+1} R_{m}^{\mathrm{s}} \operatorname{Pr}\left\{\vartheta_{m} \leq \frac{P g^{\mathrm{ss}}}{P^{\mathrm{p}} g^{\mathrm{ss}}+\sigma^{2}}<\vartheta_{m-1}\right\} \\
& =\sum_{m=1}^{M+1} R_{m}^{\mathrm{s}} \int_{0}^{\infty} f_{g^{\mathrm{ps}}}(y)\left(F_{g^{\mathrm{ss}}}\left(\frac{\left(P^{\mathrm{p}} y+\sigma^{2}\right) \vartheta_{m-1}}{P}\right)\right. \\
& \left.-F_{g^{\mathrm{ss}}}\left(\frac{\left(P^{\mathrm{p}} y+\sigma^{2}\right) \vartheta_{m}}{P}\right)\right) \mathrm{d} y \\
& =E_{g^{\mathrm{ps}}}\left\{\sum _ { m = 1 } ^ { M + 1 } R _ { m } ^ { \mathrm { s } } \left(F_{g^{\mathrm{ss}}}\left(\frac{\left(P^{\mathrm{p}} g^{\mathrm{ps}}+\sigma^{2}\right) \vartheta_{m-1}}{P}\right)\right.\right. \\
& \left.\left.-F_{g^{\mathrm{ss}}}\left(\frac{\left(P^{\mathrm{p}} g^{\mathrm{ps}}+\sigma^{2}\right) \vartheta_{m}}{P}\right)\right)\right\} .
\end{aligned}
$$

Here, $F_{g^{\mathrm{ss}}}(g)$ is the cumulative distribution function (cdf) of the SU-SU link gain and $E_{g^{\mathrm{ps}}}($.$) denotes the expectation with$ respect to the PU-SU link fading distribution. Finally, note that, with the same procedure, it is possible to extend the results to the case when there are $N>1$ primary users ${ }^{3}$. In this way, (11) is replaced by

$$
\begin{array}{r}
\bar{R}^{\mathrm{s}}=E_{g^{\mathrm{p}} 1^{\mathrm{s}}, \ldots, g^{\mathrm{p}} N^{\mathrm{s}}}\left\{\sum _ { m = 1 } ^ { M + 1 } R _ { m } ^ { \mathrm { s } } \left(F_{g^{\mathrm{ss}}}\left(\frac{\left(\sum_{i=1}^{N} P^{\mathrm{p}_{i}} g^{\mathrm{p}^{\mathrm{s}}}+\sigma^{2}\right) \vartheta_{m-1}}{P}\right)\right.\right. \\
\left.\left.-F_{g^{\mathrm{ss}}}\left(\frac{\left(\sum_{i=1}^{N} P^{\mathrm{p}_{i}} g^{\mathrm{p}_{i}^{\mathrm{s}}}+\sigma^{2}\right) \vartheta_{m}}{P}\right)\right)\right\}
\end{array}
$$

where $P^{\mathrm{p}_{i}}$ 's are corresponding transmission powers of the primary users and the expectation is taken with respect to the gains distributions $f_{g^{\mathrm{p} i}}\left(y_{i}\right), i=1 \ldots N$. As it will be seen in the following, based on the channel fading distributions, (11) and (12) can be simplified further.

Finally, it is interesting to evaluate the asymptotic performance limits of the HARQ scheme as the number of ARQ bits goes to zero or infinity; define the new variable $u_{m}=\frac{\left(P^{\mathrm{p}} g^{\mathrm{ps}}+\sigma^{2}\right) \vartheta_{m}}{P}$. Therefore, as $M \rightarrow \infty,(11)$ is changed to

$$
\begin{aligned}
\bar{R}^{\mathrm{s}, \infty} & =E_{g^{\mathrm{ps}}}\left\{\sum_{m=1}^{\infty} \log \left(1+\frac{P u_{m}}{P^{\mathrm{p}} g^{\mathrm{ps}}+\sigma^{2}}\right)\left(F_{g^{\mathrm{ss}}}\left(u_{m-1}\right)-F_{g^{\mathrm{ss}}}\left(u_{m}\right)\right)\right\} \\
& =E_{g^{\mathrm{ps}}}\left\{\int_{0}^{\infty} f_{g^{\mathrm{ss}}}(u) \log \left(1+\frac{P u}{P^{\mathrm{p}} g^{\mathrm{ps}}+\sigma^{2}}\right) \mathrm{d} u\right\} \\
& =E_{g^{\mathrm{ps}}, g^{s \mathrm{~s}}}\left\{\log \left(1+\frac{P g^{\mathrm{ss}}}{P^{\mathrm{p}} g^{\mathrm{ps}}+\sigma^{2}}\right)\right\}
\end{aligned}
$$

\footnotetext{
${ }^{3}$ The primary users considered in this part are not necessarily the license holders but are the users that, while sharing the same spectrum, are out of control for the considered secondary user.
} 
Note that (13) not only shows the ultimate limit of the proposed approach but also is an appropriate performance yardstick for delay insensitive applications.

Considering no ARQ feedback, on the other hand, a fixed rate $R_{1}^{\mathrm{s}}$ is selected and data transmission is done with no knowledge about the channel quality. The data is successfully decoded if $R_{1}^{\mathrm{s}} \leq \log \left(1+\frac{P g^{\mathrm{ss}}}{P^{\mathrm{p}} g^{\mathrm{ps}}+\sigma^{2}}\right)$. Consequently, the maximum expected rate of the channel with no knowledge at the SU transmitter is obtained by

$$
\begin{aligned}
\bar{R}^{\mathrm{s}, \text { No }} & =\max _{R_{1}^{\mathrm{s}}}\left(R_{1}^{\mathrm{s}} \operatorname{Pr}\left\{\log \left(1+\frac{P g^{\mathrm{ss}}}{P^{\mathrm{p}} g^{\mathrm{ps}}+\sigma^{2}}\right) \geq R_{1}^{\mathrm{s}}\right\}\right) \\
& =\max _{R_{1}^{\mathrm{s}}}\left(R_{1}^{\mathrm{s}}\left(1-E_{g^{\mathrm{ps}}}\left\{F_{g^{\mathrm{ss}}}\left(\frac{\left(e^{R_{1}^{\mathrm{s}}}-1\right)\left(P^{\mathrm{p}} g^{\mathrm{ps}}+\sigma^{2}\right)}{P}\right)\right\}\right)\right)
\end{aligned}
$$

which can be solved numerically.

\section{B. Long-term power constraint}

Equation (11) provides the maximum average rate of the secondary channel under the assumption that the transmission power is constant during the entire $M+1$ transmissions. Intuitively, the transmission efficiency can be improved by using optimal power allocation among the (re)transmissions. In this case, (8) should be solved in its most general case which, as mentioned before, leads to a complex optimization problem. In the following, we first study the simplest case of (8) where using 1 bit ARQ feedback the effect of power allocation on the average rate of the secondary channel is investigated both analytically and numerically. Later, the results are generalized to the case of arbitrary number of INR ARQ feedback bits.

Considering (4)-(6) and $M=1$ bit ARQ feedback, the total input power of the secondary transmitter, i.e., (7), can be rewritten as

$$
\begin{aligned}
\bar{P}^{\mathrm{s}} & =P_{1}^{\mathrm{s}} \operatorname{Pr}\left\{S_{1}\right\}+\frac{P_{1}^{\mathrm{s}} T_{1}+P_{2}^{\mathrm{s}} T_{2}}{T_{1}+T_{2}}\left(1-\operatorname{Pr}\left\{S_{1}\right\}\right) \\
& \stackrel{(a)}{=} P_{1}^{\mathrm{s}} \operatorname{Pr}\left\{S_{1}\right\}+\frac{P_{1}^{\mathrm{s}} R_{2}^{\mathrm{s}}+P_{2}^{\mathrm{s}}\left(R_{1}^{\mathrm{s}}-R_{2}^{\mathrm{s}}\right)}{R_{1}^{\mathrm{s}}}\left(1-\operatorname{Pr}\left\{S_{1}\right\}\right)
\end{aligned}
$$

in which (a) is based on (1) and $\operatorname{Pr}\left\{S_{1}\right\}$ is determined by

$$
\begin{aligned}
\operatorname{Pr}\left\{S_{1}\right\} & =\operatorname{Pr}\left\{\log \left(1+\frac{g^{\mathrm{ss}} P_{1}^{\mathrm{s}}}{g^{\mathrm{ps}} P^{\mathrm{p}}+\sigma^{2}}\right) \geq R_{1}^{\mathrm{s}}\right\} \\
& =\int_{0}^{\infty} f_{g^{\mathrm{ps}}}(y) \operatorname{Pr}\left\{g^{\mathrm{ss}} \geq \frac{\vartheta_{1}\left(P^{\mathrm{p}} y+\sigma^{2}\right)}{P_{1}^{\mathrm{s}}}\right\} \mathrm{d} y \\
& =1-E_{g^{\mathrm{ps}}}\left(F_{g^{\mathrm{ss}}}\left(\frac{\vartheta_{1}\left(P^{\mathrm{p}} g^{\mathrm{ps}}+\sigma^{2}\right)}{P_{1}^{\mathrm{s}}}\right)\right) .
\end{aligned}
$$

Moreover, using (1) and (2), the average rate of the secondary channel is found as

$$
\begin{aligned}
\bar{R}^{\mathrm{s}} & =R_{1}^{\mathrm{s}} \operatorname{Pr}\left\{\log \left(1+\frac{g^{\mathrm{ss}} P_{1}^{\mathrm{s}}}{g^{\mathrm{ps}} P^{\mathrm{p}}+\sigma^{2}}\right) \geq R_{1}^{\mathrm{s}}\right\} \\
& +R_{2}^{\mathrm{s}} \operatorname{Pr}\left\{\log \left(1+\frac{g^{\mathrm{ss}} P_{1}^{\mathrm{s}}}{g^{\mathrm{ps}} P^{\mathrm{p}}+\sigma^{2}}\right)<R_{1}^{\mathrm{s}} \&\right. \\
& \left.\frac{T_{1}}{T_{1}+T_{2}} \log \left(1+\frac{g^{\mathrm{ss}} P_{1}^{\mathrm{s}}}{g^{\mathrm{ps}} P^{\mathrm{p}}+\sigma^{2}}\right)+\frac{T_{2}}{T_{1}+T_{2}} \log \left(1+\frac{g^{\mathrm{ss}} P_{2}^{\mathrm{s}}}{g^{\mathrm{ps}} P^{\mathrm{p}}+\sigma^{2}}\right) \geq R_{2}^{\mathrm{s}}\right\} \\
& =R_{1}^{\mathrm{s}} \operatorname{Pr}\left\{\log \left(1+\frac{g^{\mathrm{s}} P_{1}^{\mathrm{s}}}{g^{\mathrm{s}} P^{P^{\mathrm{p}}+\sigma^{2}}}\right) \geq R_{1}^{\mathrm{s}}\right\} \\
& +R_{2}^{\mathrm{s}} \operatorname{Pr}\left\{\log \left(1+\frac{g^{\mathrm{s}} P_{1}^{\mathrm{s}}}{g^{\mathrm{s}} P^{\mathrm{p}}+\sigma^{2}}\right)<R_{1}^{\mathrm{s}} \&\right. \\
& \left.\frac{R_{2}^{\mathrm{s}}}{R_{1}^{\mathrm{s}}} \log \left(1+\frac{g^{\mathrm{ss}} P_{1}^{\mathrm{s}}}{g^{\mathrm{ps}} P^{\mathrm{p}}+\sigma^{2}}\right)+\frac{R_{1}^{\mathrm{s}}-R_{2}^{\mathrm{s}}}{R_{1}^{\mathrm{s}}} \log \left(1+\frac{g^{\mathrm{ss}} P_{2}^{\mathrm{s}}}{g^{\mathrm{ps}} P^{\mathrm{p}}+\sigma^{2}}\right) \geq R_{2}^{\mathrm{s}}\right\}
\end{aligned}
$$

which can be rewritten as

$$
\begin{aligned}
\bar{R}^{\mathrm{s}} & =R_{1}^{\mathrm{s}} \int_{0}^{\infty} f_{g^{\mathrm{ps}}}(y)\left(1-F_{g^{\mathrm{ss}}}\left(\frac{\vartheta_{1}\left(P^{\mathrm{p}} y+\sigma^{2}\right)}{P_{1}^{\mathrm{s}}}\right)\right) \mathrm{d} y \\
& +R_{2}^{\mathrm{s}} \int_{0}^{\infty} f_{g^{\mathrm{ps}}}(y) \theta(y) \mathrm{d} y .
\end{aligned}
$$

Here, $\theta(y)$ is defined as

$$
\theta(y) \doteq \int_{0}^{\frac{\vartheta_{1}}{P_{1}^{\mathrm{s}}}\left(P^{\mathrm{p}} y+\sigma^{2}\right)} f_{g^{\mathrm{ss}}}(t) I\left\{\varphi(t), R_{2}^{\mathrm{s}}\right\} \mathrm{d} t
$$

in which we have

$$
I\{u, v\} \doteq\left\{\begin{array}{l}
1 \text { if } u \geq v \\
0 \text { if } u<v
\end{array}\right.
$$

and

$\varphi(t) \doteq \frac{R_{2}^{\mathrm{s}}}{R_{1}^{\mathrm{s}}} \log \left(1+\frac{t P_{1}^{\mathrm{s}}}{y P^{\mathrm{p}}+\sigma^{2}}\right)+\frac{R_{1}^{\mathrm{s}}-R_{2}^{\mathrm{s}}}{R_{1}^{\mathrm{s}}} \log \left(1+\frac{t P_{2}^{\mathrm{s}}}{y P^{\mathrm{p}}+\sigma^{2}}\right)$.

In this way, defining $c(y) \doteq \arg \left\{\varphi(t)=R_{2}^{\text {s }}\right\}$, we can use (18) and (19) to show that

$$
\begin{aligned}
\bar{R}^{\mathrm{s}}=R_{1}^{\mathrm{s}} \int_{0}^{\infty} f_{g^{\mathrm{ps}}}(y)\left(1-F_{g^{\mathrm{ss}}}\left(\frac{\vartheta_{1}\left(P^{\mathrm{p}} y+\sigma^{2}\right)}{P_{1}^{\mathrm{s}}}\right)\right) \mathrm{d} y \\
+R_{2}^{\mathrm{s}} \int_{0}^{\infty} f_{g^{\mathrm{ps}}}(y)\left\{F_{g^{\mathrm{ss}}}\left(\frac{\vartheta_{1}\left(P^{\mathrm{p}} y+\sigma^{2}\right)}{P_{1}^{\mathrm{s}}}\right)-F_{g^{\mathrm{ss}}}(c(y))\right\} \mathrm{d} y \\
=E_{g^{\mathrm{ps}}}\left\{R_{1}^{\mathrm{s}}\left(1-F_{g^{\mathrm{ss}}}\left(\frac{\vartheta_{1}\left(P^{\mathrm{p}} g^{\mathrm{ps}}+\sigma^{2}\right)}{P_{1}^{\mathrm{s}}}\right)\right)\right. \\
\left.\quad+R_{2}^{\mathrm{s}}\left(F_{g^{\mathrm{ss}}}\left(\frac{\vartheta_{1}\left(P^{\mathrm{p}} g^{\mathrm{ps}}+\sigma^{2}\right)}{P_{1}^{\mathrm{s}}}\right)-F_{g^{\mathrm{ss}}}\left(c\left(g^{\mathrm{ps}}\right)\right)\right)\right\} .
\end{aligned}
$$

Finally, note that, since $R_{1}^{\mathrm{s}} \geq R_{2}^{\mathrm{s}}$ it is easy to show that $\varphi(0) \leq R_{2}^{\mathrm{s}} \leq \varphi\left(\frac{\vartheta_{1}\left(P^{\mathrm{p}} y+\sigma^{2}\right)}{P_{1}^{\mathrm{s}}}\right)$ and $\varphi(t)$ is an increasing function of $t$. Therefore, $c(y)$ will be unique for any value of $R_{2}^{\mathrm{s}}$. Unfortunately, to the best of author's knowledge, there is no closed form solution for $c(y)$. Therefore, we propose an approximate solution of (22) in the low SINR regime as follows.

Considering high $\left(P^{\mathrm{p}}, \sigma^{2}\right)$, we have $\log \left(1+\frac{g^{\mathrm{ss}} P_{k}^{\mathrm{s}}}{y P^{\mathrm{p}}+\sigma^{2}}\right) \approx$ $\frac{g^{\mathrm{ss}} P_{k}^{\mathrm{s}}}{y P^{\mathrm{p}}+\sigma^{2}}$. Therefore, one can use (21) to find the approximate values of $c(y)$ and $\bar{R}^{\mathrm{s}}$ as

$$
c(y) \approx \frac{R_{1}^{\mathrm{s}} R_{2}^{\mathrm{s}}}{R_{2}^{\mathrm{s}} P_{1}^{\mathrm{s}}+\left(R_{1}^{\mathrm{s}}-R_{2}^{\mathrm{s}}\right) P_{2}^{\mathrm{s}}}\left(P^{\mathrm{p}} y+\sigma^{2}\right)
$$

and

$$
\begin{aligned}
& \bar{R}^{\mathrm{s}} \approx E_{g^{\mathrm{ps}}}\left\{R_{1}^{\mathrm{s}}\left(1-F_{g^{\mathrm{ss}}}\left(\frac{\vartheta_{1}\left(P^{\mathrm{p}} g^{\mathrm{ps}}+\sigma^{2}\right)}{P_{1}^{\mathrm{s}}}\right)\right)+\right. \\
& \left.R_{2}^{\mathrm{s}}\left(F_{g^{\mathrm{ss}}}\left(\frac{\vartheta_{1}\left(P^{\mathrm{p}} g^{\mathrm{ps}}+\sigma^{2}\right)}{P_{1}^{\mathrm{s}}}\right)-F_{g^{\mathrm{ss}}}\left(\frac{R_{1}^{\mathrm{s}} R_{2}^{\mathrm{s}}}{R_{2}^{\mathrm{s}} P_{1}^{\mathrm{s}}+\left(R_{1}^{\mathrm{s}}-R_{2}^{\mathrm{s}}\right) P_{2}^{\mathrm{s}}}\left(P^{\mathrm{p}} g^{\mathrm{ps}}+\sigma^{2}\right)\right)\right)\right\}
\end{aligned}
$$

respectively. Note that, based on the fact that $\log (1+x) \leq$ $x, x>0$, the above approximation has increased the second probability term of (17) (or equivalently $\theta(y)$ ) and so (24) gives an upper bound of secondary channel average rates. Then, with the same procedure, the results can be extended 
to the case of arbitrary number of ARQ feedback bits; using (5), (4) changes to

$$
\begin{aligned}
& \operatorname{Pr}\left\{S_{m}\right\}=\int_{0}^{\infty} f_{g^{\mathrm{ps}}}(y) \times \\
& \quad \operatorname{Pr}\left\{\sum_{k=1}^{m} \frac{T_{k}}{\sum_{j=1}^{m} T_{j}} \log \left(1+\frac{P_{k}^{\mathrm{s}} g^{\mathrm{ss}}}{P^{\mathrm{p}} y+\sigma^{2}}\right) \geq R_{m}^{\mathrm{s}}\right\} \mathrm{d} y \\
& \left.\approx \int_{0}^{\infty} f_{g^{\mathrm{ps}}}(y) \operatorname{Pr}\left\{g^{\mathrm{ss}} \sum_{k=1}^{m} \frac{T_{k} P_{k}^{\mathrm{s}}}{\sum_{j=1}^{m} T_{j}}\right) \geq R_{m}^{\mathrm{s}}\left(P^{\mathrm{p}} y+\sigma^{2}\right)\right\} \mathrm{d} y \\
& =E_{g^{\mathrm{ps}}}\left\{1-F_{g^{\mathrm{ss}}}\left(\frac{P^{\mathrm{p}} y+\sigma^{2}}{w_{m}}\right)\right\}
\end{aligned}
$$

where $w_{m} \doteq \sum_{k=1}^{m} P_{k}^{\mathrm{s}}\left(\frac{1}{R_{k}^{\mathrm{s}}}-\frac{1}{R_{k-1}^{\mathrm{s}}}\right), w_{0}=0$. Hence, the rate optimization problem (8) is simplified to

$$
\bar{R}^{\mathrm{s}, \max } \approx \max _{\left\{P_{m}^{\mathrm{s}}, R_{m}^{\mathrm{s}}, m=1 \ldots M+1\right\}} E_{g^{\mathrm{ps}}}\left\{\sum _ { m = 1 } ^ { M + 1 } R _ { m } ^ { \mathrm { s } } \left(F_{g^{\mathrm{ss}}}\left(\frac{P^{\mathrm{p}} g^{\mathrm{ps}}+\sigma^{2}}{w_{m-1}}\right)\right.\right.
$$$$
\begin{aligned}
&\left.\left.-F_{g^{\mathrm{ss}}}\left(\frac{P^{\mathrm{p}} g^{\mathrm{ps}}+\sigma^{2}}{w_{m}}\right)\right)\right\} \\
& \text { subject to } E_{g^{\mathrm{ps}}}\left\{\sum_{m=1}^{M} P_{(m)}^{\mathrm{s}}\left(F_{g^{\mathrm{ss}}}\left(\frac{P^{\mathrm{p}} g^{\mathrm{ps}}+\sigma^{2}}{w^{2}-1}\right)-F_{g^{\mathrm{ss}}}\left(\frac{P^{\mathrm{p}} g^{\mathrm{ps}}+\sigma^{2}}{w_{m}}\right)\right)\right.
\end{aligned}
$$$$
\left.\left.+P_{(M+1)}^{\mathrm{s}} F_{g^{\mathrm{ss}}}\left(\frac{P^{\mathrm{p}} g^{\mathrm{ps}}+\sigma^{2}}{w_{M}}\right)\right)\right\} \leq P
$$

which can be solved numerically. Finally, it is worth noting that, one can use $\log (1+x) \approx \log (x)$ to simplify the probability term $\operatorname{Pr}\left\{S_{m}\right\}$ into

$$
\begin{aligned}
& \operatorname{Pr}\left\{S_{m}\right\} \approx \int_{0}^{\infty} f_{g^{\mathrm{ps}}}(y) \times \\
& \quad \operatorname{Pr}\left\{\sum_{k=1}^{m} \frac{T_{k}}{\sum_{j=1}^{m} T_{j}} \log \left(\frac{P_{k}^{\mathrm{s}} g^{\mathrm{ss}}}{P^{\mathrm{p}} y+\sigma^{2}}\right) \geq R_{m}^{\mathrm{s}}\right\} \mathrm{d} y \\
&= \int_{0}^{\infty} f_{g^{\mathrm{ps}}}(y) \operatorname{Pr}\left\{\log \left(\frac{g^{\mathrm{ss}}}{P^{\mathrm{p}} y+\sigma^{2}}\right)\right. \\
& \quad\left.\quad R_{m}^{\mathrm{s}} \sum_{k=1}^{m}\left(\frac{1}{R_{k}^{\mathrm{s}}}-\frac{1}{R_{k-1}^{\mathrm{s}}}\right) \log \left(P_{k}^{\mathrm{s}}\right) \geq R_{m}^{\mathrm{s}}\right\} \mathrm{d} y \\
&= E_{g^{\mathrm{ps}}}\left\{1-F_{g^{\mathrm{ss}}}\left(\left(P^{\mathrm{p}} g^{\mathrm{ps}}+\sigma^{2}\right) q_{m}\right)\right\}, \\
& q_{m}=e^{R_{m}^{\mathrm{s}}-R_{m}^{\mathrm{s}} \log \prod_{k=1}^{m}\left(P_{k}^{\mathrm{s}}\right)^{\frac{1}{R_{k}^{\mathrm{s}}}}-\frac{1}{R_{k-1}^{\mathrm{s}}}}, q_{0}=\infty
\end{aligned}
$$

which can be used for rate approximation under high SINR regime. However, since the bound is not tight for the considered distributions and also because the effect of optimal power allocation diminishes as the transmission power increases [27]-[30], we do not discuss it any further.

\section{Special case; Rayleigh-fading channel}

This part presents the secondary channel average rates for Rayleigh-fading channels, e.g., $f_{g^{\mathrm{ss}}}(x)=\frac{1}{\lambda^{\mathrm{ss}}} e^{-\frac{x}{\lambda^{\mathrm{ss}}}}, x \geq 0$, where $\lambda^{\mathrm{sp}}, \lambda^{\mathrm{ps}}, \lambda^{\mathrm{pp}}$ and $\lambda^{\mathrm{ss}}$ denote the corresponding means of exponential pdfs normally determined by the path loss and shadowing between the terminals. In this way, considering short-term power allocation the average rate of the secondary channel, i.e., (11), is found as

$$
\begin{aligned}
& \bar{R}^{\mathrm{s}}=\sum_{m=1}^{M+1} R_{m}^{\mathrm{s}}\left(\int _ { 0 } ^ { \infty } \frac { 1 } { \lambda ^ { \mathrm { ps } } } e ^ { - \frac { y } { \lambda ^ { \mathrm { Ps } } } } \left(e^{-\frac{\vartheta_{m}\left(P^{\mathrm{p}} y+\sigma^{2}\right)}{\lambda^{\mathrm{ss} P}}}\right.\right. \\
& -e^{\left.\left.-\frac{\vartheta_{m-1}\left(P^{\mathrm{p}} y+\sigma^{2}\right)}{\lambda^{\mathrm{ss} P}}\right)\right) \mathrm{d} y} \\
& =\sum_{m=1}^{M+1} R_{m}^{\mathrm{s}}\left(\frac{e^{-\frac{\sigma^{2} \vartheta_{m}}{\lambda^{\mathrm{ss} P}}}}{1+\frac{\lambda^{\mathrm{S} P \mathrm{P}} \vartheta_{m}}{\lambda^{\mathrm{s} P} P}}-\frac{e^{-\frac{\sigma^{2} \vartheta_{m}-1}{\lambda^{\mathrm{s} P} P}}}{1+\frac{\lambda^{\mathrm{PS} P} \vartheta_{m-1}}{\lambda^{\mathrm{ss} P}}}\right) .
\end{aligned}
$$

Moreover, considering $N>1$ primary transmitters with powers $P^{\mathrm{p}_{i}}$ and Rayleigh-fading channels having parameters $\lambda^{\mathrm{p}_{i} \mathrm{~s}}=\lambda_{i},(12)$ is found as

$$
\bar{R}^{\mathrm{s}}=\sum_{m=1}^{M+1} R_{m}^{\mathrm{s}}\left(\frac{e^{-\frac{\sigma^{2} \vartheta_{m}}{\lambda^{\mathrm{ss} P}}}}{\prod_{i=1}^{N}\left(1+\frac{\lambda_{i} P^{\mathrm{p}_{i} \vartheta_{m}}}{\lambda^{\mathrm{ss} P}}\right)}-\frac{e^{-\frac{\sigma^{2} \vartheta_{m-1}}{\lambda^{\mathrm{ss} P}}}}{\prod_{i=1}^{N}\left(1+\frac{\lambda_{i} P^{\mathrm{p}_{i} \vartheta_{m-1}}}{\lambda^{\mathrm{ss} P}}\right)}\right.
$$

Interestingly, assuming infinitely many, i.e., $N \rightarrow \infty$, users with properties $P^{\mathrm{p}_{i}}=\frac{P}{N}$ and $\lambda_{i}=\lambda^{\mathrm{ss}},(27)$ is simplified to

$$
\bar{R}^{\mathrm{s}}=\sum_{m=1}^{M+1} R_{m}^{\mathrm{s}}\left(e^{-\left(\frac{\sigma^{2}+\lambda^{\mathrm{ss}} P}{\lambda^{\mathrm{ss}} P}\right) \vartheta_{m}}-e^{-\left(\frac{\sigma^{2}+\lambda^{\mathrm{ss}} P}{\lambda^{\mathrm{ss}} P}\right) \vartheta_{m-1}}\right) .
$$

Implementing infinitely many HARQ rounds, on the other hand, (13) is simplified to

$$
\begin{aligned}
\bar{R}^{\mathrm{s}, \infty} & =\int_{0}^{\infty} \frac{1}{\lambda^{\mathrm{ps}}} \frac{1}{\lambda^{\mathrm{ss}}} e^{-\frac{y}{\lambda^{\mathrm{ps}}}} \int_{0}^{\infty} e^{-\frac{x}{\lambda^{\mathrm{ss}}}} \log \left(1+\frac{P x}{P^{\mathrm{p}} y+\sigma^{2}}\right) \mathrm{d} x \mathrm{~d} y \\
& =-\frac{1}{\lambda^{\mathrm{ps}}} e^{-\frac{\sigma^{2}}{\lambda^{\mathrm{ss} P}}} \int_{0}^{\infty} e^{-\left(\frac{1}{\lambda^{\mathrm{ps}}}-\frac{P \mathrm{p}}{\lambda^{\mathrm{ss} P}}\right) y} \operatorname{Ei}\left(-\frac{P^{\mathrm{p}}}{\lambda^{\mathrm{ss}} P} y-\frac{\sigma^{2}}{\lambda^{\mathrm{ss} P}}\right) \mathrm{d} y \\
& =\frac{1}{\frac{\lambda^{\mathrm{ps} P}}{\lambda^{\mathrm{ss} P}}-1}\left(e^{\frac{\sigma^{2}}{\lambda^{\mathrm{ss}} P}} \operatorname{Ei}\left(-\frac{\sigma^{2}}{\lambda^{\mathrm{ss} P}}\right)-e^{\frac{\sigma^{2}}{\lambda^{\mathrm{ps}} P^{\mathrm{p}}}} \operatorname{Ei}\left(-\frac{\sigma^{2}}{\lambda^{\mathrm{s}} P^{\mathrm{p}}}\right)\right)
\end{aligned}
$$

where $\operatorname{Ei}($.$) is the standard exponential integral function.$ Finally, considering long-term power allocation the 1-bit lowSINR average rate of the secondary link and its average input power, i.e., (15), are obtained by

$$
\begin{aligned}
\bar{R}^{\mathrm{s}} & \leq \frac{R_{1}^{\mathrm{s}} e^{-\frac{\vartheta_{1} \sigma^{2}}{\lambda^{\mathrm{ss}} P_{1}^{\mathrm{s}}}}}{1+\frac{\lambda^{\mathrm{ps}} \vartheta_{1} P^{\mathrm{p}}}{\lambda^{\mathrm{ss}} P_{1}^{\mathrm{s}}}}+\frac{R_{2}^{\mathrm{s}} e^{-\frac{\eta \sigma^{2}}{\lambda^{\mathrm{ss}} P_{1}^{\mathrm{s}}}}}{1+\frac{\lambda^{\mathrm{p} s} \eta P^{\mathrm{p}}}{\lambda^{\mathrm{ss}} P_{1}^{\mathrm{s}}}}-\frac{R_{2}^{\mathrm{s}} e^{-\frac{\vartheta_{1} \sigma^{2}}{\lambda^{\mathrm{ss}} P_{1}^{\mathrm{s}}}}}{1+\frac{\lambda^{\mathrm{ps}} \vartheta_{1} P^{\mathrm{p}}}{\lambda^{\mathrm{ss}} P_{1}^{\mathrm{s}}}} \\
\eta & =\frac{R_{1}^{\mathrm{s}} R_{2}^{\mathrm{s}} P_{1}^{\mathrm{s}}}{R_{2}^{\mathrm{s}} P_{1}^{\mathrm{s}}+\left(R_{1}^{\mathrm{s}}-R_{2}^{\mathrm{s}}\right) P_{2}^{\mathrm{s}}}
\end{aligned}
$$

and

$\bar{P}^{\mathrm{s}}=\frac{P_{1}^{\mathrm{s}} e^{-\frac{\vartheta_{1} \sigma^{2}}{\lambda^{\mathrm{ss}} P_{1}^{\mathrm{s}}}}}{1+\frac{\lambda^{\mathrm{p} s} \vartheta_{1} P^{\mathrm{p}}}{\lambda^{\mathrm{s}} P_{1}^{\mathrm{s}}}}+\frac{R_{2}^{\mathrm{s}} P_{1}^{\mathrm{s}}+\left(R_{1}^{\mathrm{s}}-R_{2}^{\mathrm{s}}\right) P_{2}^{\mathrm{s}}}{R_{1}^{\mathrm{s}}}\left(1-\frac{e^{-\frac{\vartheta_{1} \sigma^{2}}{\lambda^{\mathrm{s}} P_{1}^{\mathrm{s}}}}}{1+\frac{\lambda^{\mathrm{p}} \vartheta_{1} P^{\mathrm{p}}}{\lambda^{\mathrm{ss}} P_{1}^{\mathrm{s}}}}\right)$

respectively. Implementing Lagrange multiplier or numerical methods, it is possible to find the optimal values of $P_{k}^{\mathrm{s}}, R_{k}^{\mathrm{s}}, k=1,2$ maximizing the channel average rate under $\bar{P}^{\mathrm{s}} \leq P$ constraint

Equations (26)-(31) are studied in more detail through figures 2-5; considering short-term power constraint and different number of ARQ feedback bits, Fig. 2 and 3 show the average rate of the SU-SU channel for fixed PU and SU transmission powers, respectively. Also, the figures present the results obtained under no knowledge, i.e., (14), and $M \rightarrow \infty$ assumptions as two lower and upper bounds, respectively. To evaluate the effect of different fading distributions, we have also obtained the results for Rayleigh gain pdfs, e.g., $f_{g^{\mathrm{ss}}}(x)=\frac{x}{\lambda^{\mathrm{ss}}} e^{-\frac{x^{2}}{2 \lambda^{\mathrm{ss}}}}, x \geq 0$, as illustrated in Fig. 2 and $3 .{ }^{4}$ Then, Fig. 4 investigates the effect of ARQ feedback on the SU-SU channel average rate in the presence of different number of primary users each one having power $P^{\mathrm{p}_{i}}=\frac{P}{N}$. As illustrated by the figure, with the same total primary transmission power, increasing the number of primary users can drastically reduce the SU-SU channel average rate. Finally, considering 1 bit ARQ feedback, Fig. 5 shows the upper bound

${ }^{4}$ Considering $\lambda^{\mathrm{ss}}$ and $\lambda^{\mathrm{ps}}$ as the SU-SU and PU-SU links Rayleigh pdf parameters, respectively, and the standard error function $\operatorname{erf}(x)=$ $\frac{2}{\sqrt{\pi}} \int_{0}^{x} e^{-\frac{t^{2}}{2}} \mathrm{~d} t$, we have $\operatorname{Pr}\left\{\log \left(1+\frac{P g^{\mathrm{ss}}}{P^{\mathrm{p}} \mathrm{g}^{\mathrm{p}}+\sigma^{2}}\right) \leq x\right\}=\frac{1}{2} \xi_{1} e^{-\xi_{3}}+$

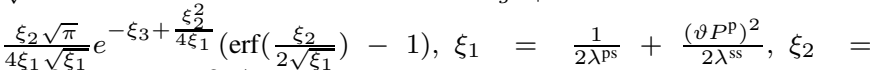
$\frac{\vartheta^{2} \sigma^{2} P^{\mathrm{p}}}{\lambda^{\mathrm{ss}}}, \xi_{3}=\frac{\vartheta^{2} \sigma^{4}}{2 \lambda^{\mathrm{ss}}}, \vartheta=\frac{e^{x}-1}{P}$, which can be used in, e.g., (11). Note that as discussed in [50], Rayleigh distributions are appropriate models for the cases where the fading is not so severe. 


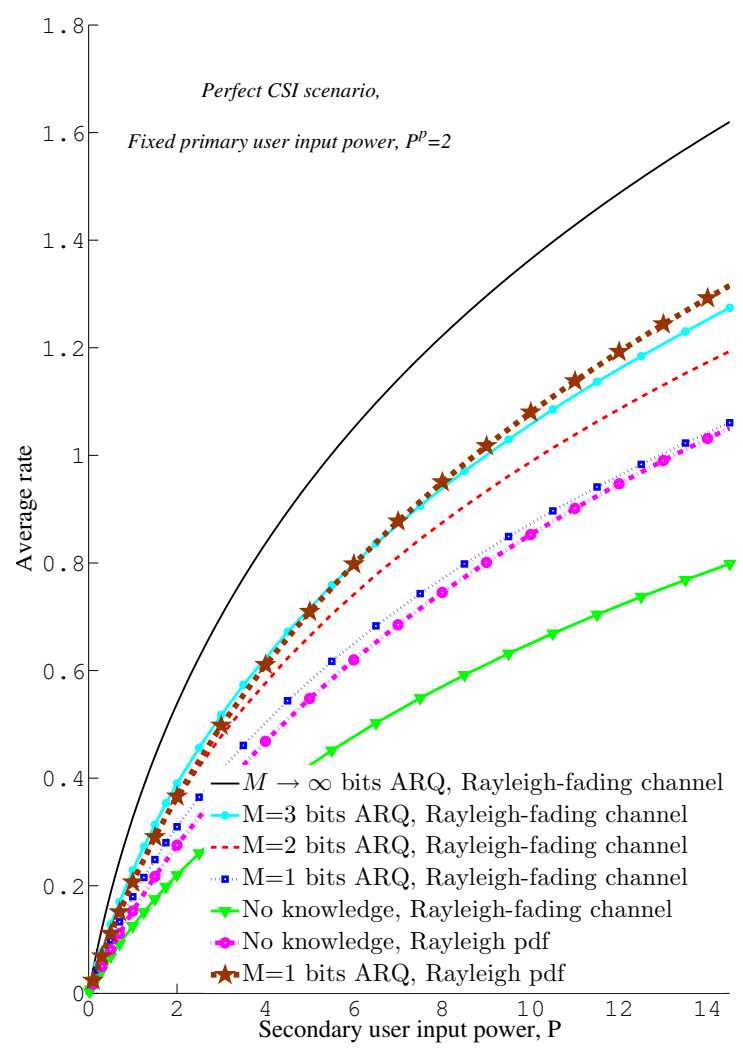

Figure 2. Average rate vs secondary user input power, perfect CSI scenario, $P^{\mathrm{p}}=2$.

of the SU-SU channel average rate, i.e., (30), under long-term power constraint. Moreover, in order to verify the tightness of the proposed upper bound, we have found the average rate upper bound under short-term $\left(P_{m}^{\mathrm{s}}=P, \forall m=1,2\right)$ condition which is compared with its exact value obtained by (26). It is worth noting that, in all simulations, the parameters of the distributions are set to 1 . Also, all results, except Fig. 5 that is found under $\sigma^{2}=10$ condition, have been obtained with noise variance $\sigma^{2}=1$. Further discussions about the simulation results are presented in Section VI.

\section{Secondary Channel aVERAGe Rate optimization; LIMITED PU RECEIVED INTERFERENCE POWER AND IMPERFECT SU-PU CSI FEEDBACK}

As mentioned before, depending on the fading characteristics such as the channel coherence time and the amount of feedback resources, it may not be possible to provide the secondary transmitter with perfect SU-PU channel state information. Hence, this part restudies the secondary channel average rate under the more realistic case where there is imperfect SU-PU CSI available at the SU transmitter. Again, the imperfect information can be obtained in different ways such as by direct collaboration of the secondary transmitter and the primary receiver or by means of a band manager which mediates between the two parties [51]. Implementing this partial channel quality information, we have an estimate of the channel which can be modeled as

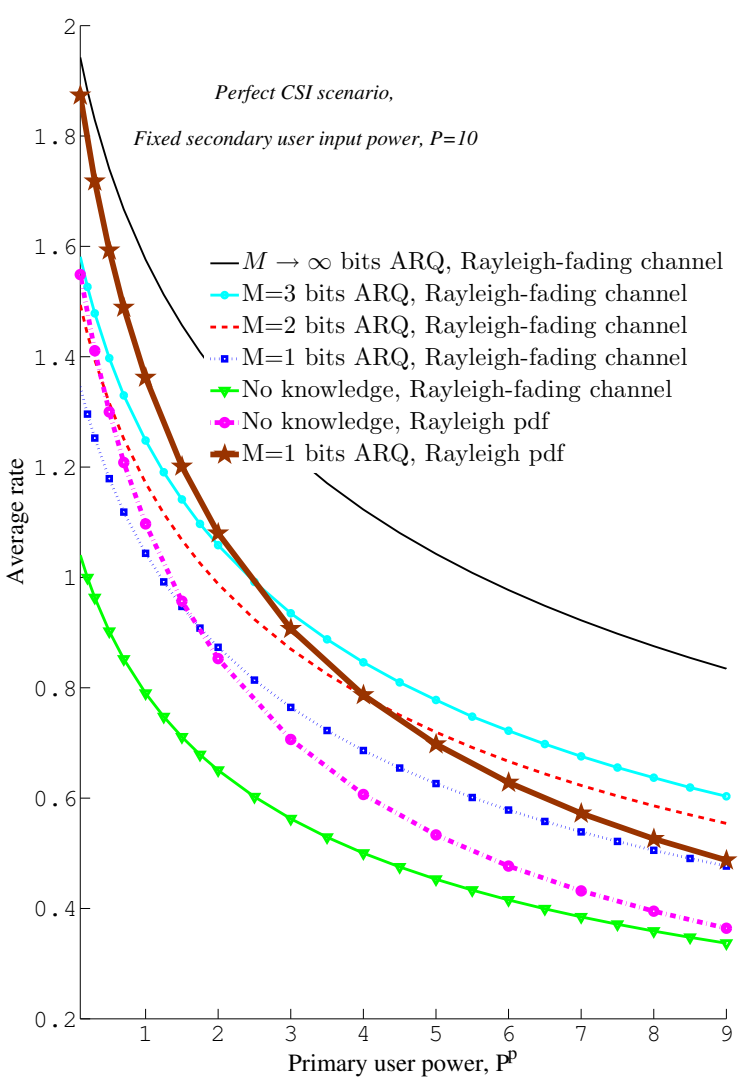

Figure 3. Average rate vs primary user input power, perfect CSI scenario, $P=10$.

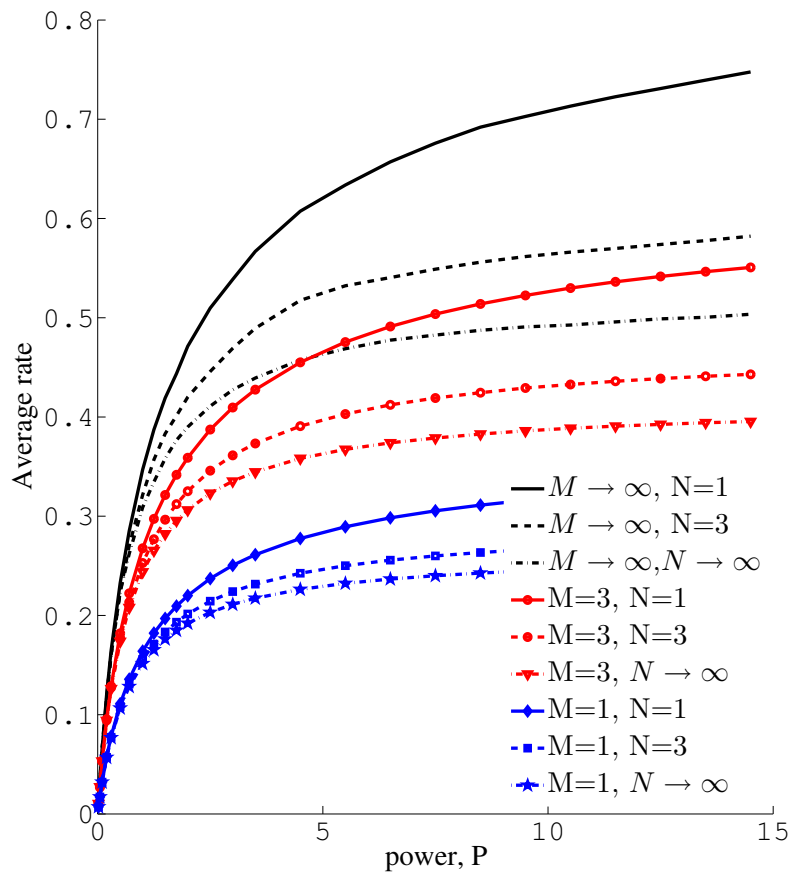

Figure 4. Average rate for different number of primary users and ARQ feedback bits, perfect CSI scenario. Each primary user has power $P^{\mathrm{p}_{i}}=\frac{P}{N}$ where $P$ is the secondary user transmission power in different ARQ rounds and $N$ is the number of primary users. 


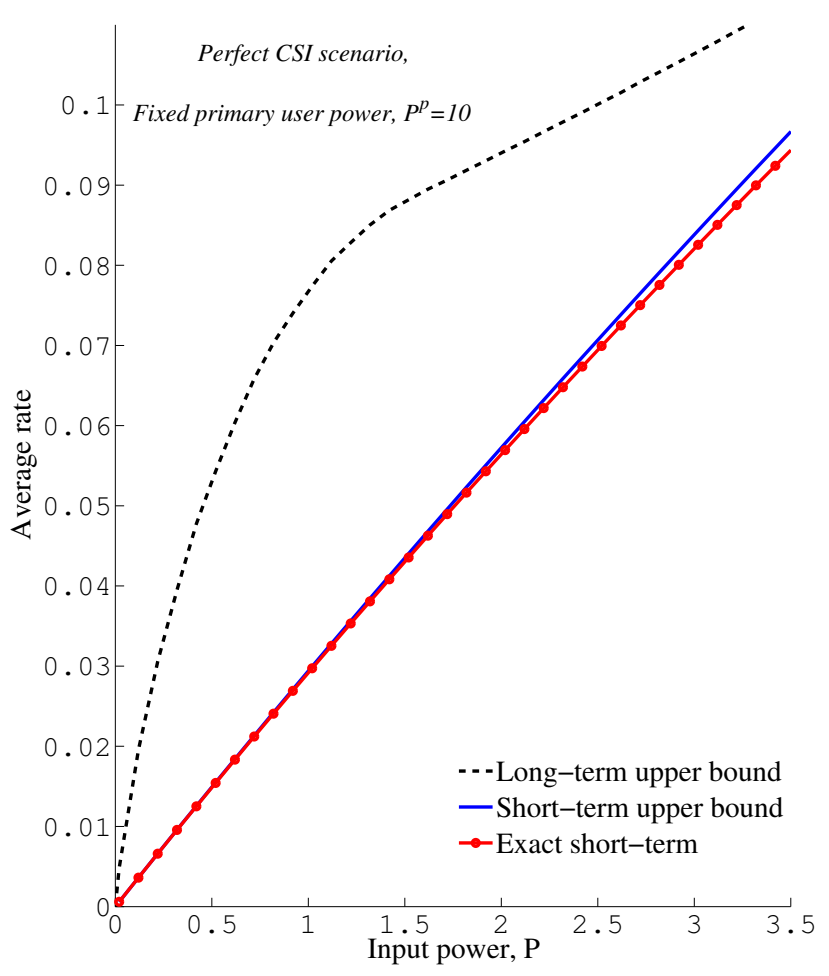

Figure 5. Secondary channel performance with one bit ARQ feedback under different power allocation strategies, perfect CSI scenario. $P^{\mathrm{p}}=10, \sigma^{2}=10$.

$$
\tilde{h}^{\mathrm{sp}}=\beta h^{\mathrm{sp}}+\sqrt{1-\beta^{2}} \varepsilon .
$$

Here, $\varepsilon$ is a Gaussian noise $\mathcal{C N}\left(0, \lambda^{\mathrm{sp}}\right)$ uncorrelated with $h^{\mathrm{sp}}$, $\tilde{h}^{\mathrm{sp}}$ represents the channel estimate available at the secondary transmitter and $\beta$ is the correlation coefficient determining the channel estimation quality. This is a well accepted model of partial channel quality information addressed in the literature [23], [52]-[54]. In practice, $\beta$ depends on a number of parameters such as training sequence, SNR, Doppler frequency or the number of quantization regions considered for the SU-PU CSI feedback [23], [52]-[54]. Finally, using (32) and the Rayleighfading channels, the probability density functions $f_{g^{\text {sp }}, \tilde{g}^{\text {sp }}}$ and $f_{\tilde{g}^{\mathrm{sp}}}, \tilde{g}^{\mathrm{sp}}=\left|\tilde{h}^{\mathrm{sp}}\right|^{2}$, are found as

$$
f_{g^{\mathrm{sp}}, \tilde{g}^{\mathrm{sp}}}(x, y)=\frac{1}{\left(1-\beta^{2}\right) \lambda^{\mathrm{sp}}{ }^{2}} e^{-\frac{x+y}{\left(1-\beta^{2}\right) \lambda^{\mathrm{sp}}}} I_{0}\left(\frac{2 \beta \sqrt{x y}}{\left(1-\beta^{2}\right) \lambda^{\mathrm{sp}}}\right)
$$

and

$$
f_{\tilde{g}^{\mathrm{sp}}}(x)=\frac{1}{\lambda^{\mathrm{sp}}} e^{-\frac{x}{\lambda^{\mathrm{sp}}}}, x \geq 0,
$$

respectively [23]. Here, $I_{0}($.$) is the zeroth-order modified$ Bessel function of the first kind.

Let $I^{\mathrm{p}}$ be the maximum peak interference that the primary receiver can tolerate. Based on the channel estimate, the transmission power of the secondary user is selected to be $P_{m}^{\mathrm{s}}=\frac{I^{\mathrm{p}}}{\tilde{g}_{\mathrm{sp}}}, m=1 \ldots M+1$. In this way, the secondary channel SINR, i.e., (3), is replaced by

$$
\gamma_{m}^{\mathrm{s}}=\frac{I^{\mathrm{p}} g^{\mathrm{ss}}}{\tilde{g}^{\mathrm{sp}}\left(P^{\mathrm{p}} g^{\mathrm{ps}}+\sigma^{2}\right)}
$$

Therefore, (10) is changed into

$$
\bar{R}^{\mathrm{s}}=\sum_{m=1}^{M+1} R_{m}^{\mathrm{s}} \operatorname{Pr}\left\{R_{m}^{\mathrm{s}} \leq \log \left(1+\frac{I^{\mathrm{p}} g^{\mathrm{ss}}}{\tilde{g}^{\mathrm{sp}}\left(P^{\mathrm{p}} g^{\mathrm{ps}}+\sigma^{2}\right)}\right)<R_{m-1}^{\mathrm{s}}\right\}
$$

which can be further simplified to

$$
\begin{gathered}
\bar{R}^{\mathrm{s}}=\sum_{m=1}^{M+1} R_{m}^{\mathrm{s}} \operatorname{Pr}\left\{\vartheta_{m} \leq \frac{I^{\mathrm{p}} g^{\mathrm{ss}}}{\tilde{g}^{\mathrm{sp}}\left(P^{\mathrm{p}} g^{\mathrm{ps}}+\sigma^{2}\right)}<\vartheta_{m-1}\right\} \\
=\sum_{m=1}^{M+1} R_{m}^{\mathrm{s}} \int_{0}^{\infty} \int_{0}^{\infty} f_{\tilde{g}^{\mathrm{sp}}}(z) f_{g^{\mathrm{ps}}}(y)\left(F_{g^{\mathrm{ss}}}\left(\frac{z\left(P^{\mathrm{p}} y+\sigma^{2}\right) \vartheta_{m}-1}{I^{\mathrm{p}}}\right)\right. \\
\left.-F_{g^{\mathrm{ss}}}\left(\frac{z\left(P^{\mathrm{p}} y+\sigma^{2}\right) \vartheta_{m}}{I^{\mathrm{p}}}\right)\right) \mathrm{d} y \mathrm{~d} z \\
=E_{\tilde{g}^{\mathrm{sp}}, g^{\mathrm{ps}}}\left\{\sum _ { m = 1 } ^ { M + 1 } R _ { m } ^ { \mathrm { s } } \left(F_{g^{\mathrm{ss}}}\left(\frac{\tilde{g}^{\mathrm{sp}}\left(P^{\mathrm{p}} g^{\mathrm{ps}}+\sigma^{2}\right) \vartheta_{m-1}}{I^{\mathrm{p}}}\right)\right.\right. \\
\left.\left.-F_{g^{\mathrm{ss}}}\left(\frac{\tilde{g}^{\mathrm{sp}}\left(P^{\mathrm{p}} g^{\mathrm{ps}}+\sigma^{2}\right) \vartheta_{m}}{I^{\mathrm{p}}}\right)\right)\right\} .
\end{gathered}
$$

Finally, considering Rayleigh-fading channels, the secondary channel average rate is obtained by

$$
\begin{aligned}
& \bar{R}^{\mathrm{s}}=\frac{1}{\lambda^{\mathrm{ps}}} \frac{1}{\lambda^{\mathrm{sp}}} \sum_{m=1}^{M+1} R_{m}^{\mathrm{s}} \int_{0}^{\infty} \int_{0}^{\infty} e^{-\left(\frac{y}{\lambda^{\mathrm{ps}}}+\frac{z}{\lambda^{\mathrm{sp}}}\right)}\left(e^{-\frac{z}{\lambda^{\mathrm{ss}} I \mathrm{p}}\left(P^{\mathrm{p}} y+\sigma^{2}\right) \vartheta_{m}}\right. \\
& \left.-e^{-\frac{z}{\lambda^{\mathrm{sS}} I \mathrm{P}}\left(P^{\mathrm{p}} y+\sigma^{2}\right) \vartheta_{m-1}}\right) \mathrm{d} y \mathrm{~d} z
\end{aligned}
$$

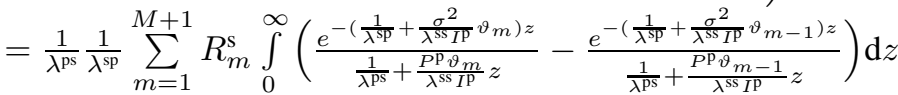

$$
\begin{aligned}
& =\frac{\lambda^{\mathrm{ss}} I^{\mathrm{p}}}{\lambda^{\mathrm{ps}} \lambda^{\mathrm{sp} P \mathrm{p}}} \sum_{m=1}^{M+1} R_{m}^{\mathrm{s}}\left(J\left(\vartheta_{m-1}\right)-J\left(\vartheta_{m}\right)\right)
\end{aligned}
$$

where

$$
J(x) \doteq \frac{e^{\left(\frac{\lambda^{\lambda^{\mathrm{ss}}} I^{\mathrm{p}}}{\lambda \mathrm{sp}_{x}}+\sigma^{2}\right) \frac{1}{\lambda^{\mathrm{ps} P \mathrm{p}}}}}{x} \operatorname{Ei}\left(-\frac{1}{\lambda^{\mathrm{ps}} P^{\mathrm{p}}}\left(\frac{\lambda^{\mathrm{ss}} I^{\mathrm{p}}}{\lambda^{\mathrm{sp}} x}+\sigma^{2}\right)\right) .
$$

Setting $P_{m}^{\mathrm{s}}=\frac{I^{\mathrm{p}}}{\tilde{g}^{\mathrm{sp}}}$, the interference power received at the primary receiver would be $\frac{g^{\mathrm{sp}} I^{\mathrm{p}}}{\tilde{g}^{\mathrm{sp}}}$. Therefore, due to imperfect secondary-primary link estimation, there may be times when the desired received interference constraint is not satisfied. In order to tackle this problem, the secondary transmitter must back off its transmission power so that it meets the primary channel quality-of-service requirements [55]. Finding the received interference cdf can help us to determine a reduced input power, so that the interference constraint is satisfied with high probability. Let $Q=\frac{g^{\text {sp }} I^{\mathrm{p}}}{\tilde{g}^{\text {sp }}}$. Then, (33) and [23, Eq. 35] can be used to find the interference cdf as

$$
\begin{aligned}
F_{\text {int }}\left(t \mid I^{\mathrm{p}}\right) & =\operatorname{Pr}\left\{Q \leq t \mid I^{\mathrm{p}}\right\}=\int_{0}^{\infty} \int_{\frac{x I^{\mathrm{p}}}{t}}^{\infty} f_{g^{\mathrm{sp}}, \tilde{g}^{\mathrm{sp}}(x, y) \mathrm{d} x \mathrm{~d} y} \\
& =\frac{1}{2}\left(1+\frac{1-\frac{I^{\mathrm{p}}}{t}}{\sqrt{\left(1+\frac{I^{\mathrm{p}}}{t}\right)^{2}-\frac{4 \beta^{2} I^{\mathrm{p}}}{t}}}\right)
\end{aligned}
$$

which is a function of the constant $I^{\mathrm{p}}$. Therefore, the new reduced power $\tilde{I}^{\mathrm{p}}$, which with probability $\pi$ keeps the interference less than $I^{\mathrm{p}}$, can be found as the solution of equation $F_{\text {int }}\left(I^{\mathrm{p}} \mid \tilde{I}^{\mathrm{p}}\right)=\pi$. Finally, with the same procedure as before, the asymptotic performance bound of the channel is obtained by

$$
\bar{R}^{\mathrm{s}, \infty}=E_{\tilde{g}^{\mathrm{sp}}, g^{\mathrm{ps}}, g^{\mathrm{ss}}}\left\{\log \left(1+\frac{I^{\mathrm{p}} g^{\mathrm{ss}}}{\tilde{g}^{\mathrm{sp}}\left(P^{\mathrm{p}} g^{\mathrm{ps}}+\sigma^{2}\right)}\right)\right\}
$$




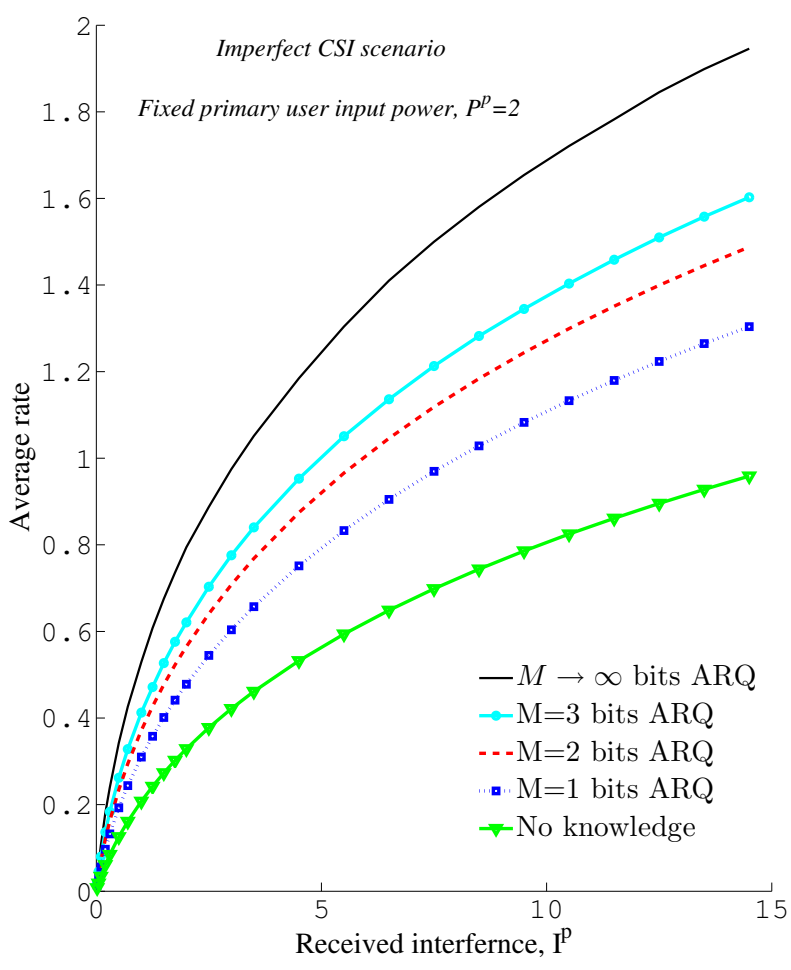

Figure 6. Average rate vs primary user received interference power, imperfect CSI scenario, $P^{\mathrm{P}}=2$.

Figures 6-9 provide better insight about the effect of ARQ feedback on the SU-SU channel performance under imperfect CSI condition; implementing different number of ARQ feedback bits, Fig. 6 and 7 demonstrate the channel average rate for fixed PU transmission and received interference powers, respectively. Again, the figures represent the two $M=0$ and $M \rightarrow \infty$ cases as two ultimate channel performance bounds. Further, setting $I^{\mathrm{p}}=10, P^{\mathrm{p}}=2$ and $\pi=0.9$, Fig. 8 verifies the influence of estimation quality, i.e., $\beta$, on the average rate of the SU-SU channel. Finally, the effect of interference probability $\pi$ is studied in Fig. 9 where the results are obtained for fixed $P^{\mathrm{p}}=2, I^{\mathrm{p}}=10$, and $\beta=0.8$.

\section{SECONDARY CHANNEL AVERAGE RATE; Limited OUTAGE PROBABILITY SCENARIO}

While the channel average rate is a parameter demonstrating the channel long-term transmission limits, outage probability is another quality-of-service metric which characterizes a network degree of reliability. There are a number of methods reducing the channel outage probability [28] among which ARQ feedback is one of the most well-known ones [6], [35], [36]. With this background, this part restudies the previous results under the condition that data transmission is constrained to have limited outage probability.

In a general ARQ-based approach outage happens if, along with all previous ones, the last retransmission fails. Therefore, considering limited peak transmission power, the outage

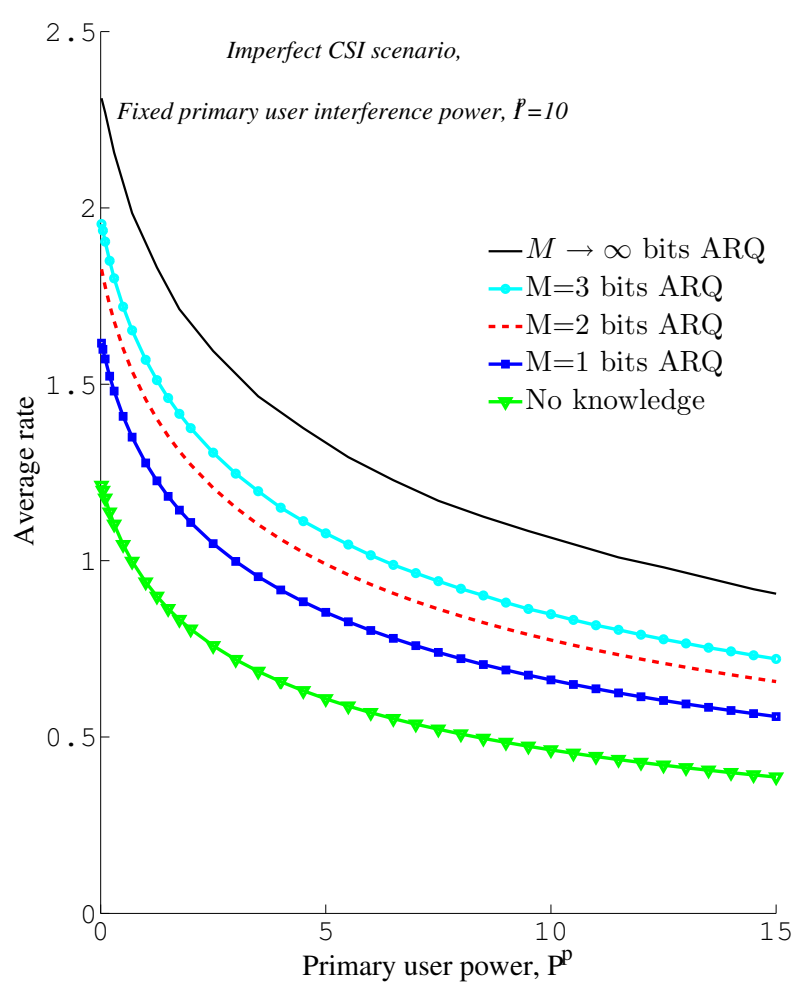

Figure 7. Average rate vs primary user input power, imperfect CSI scenario, $I^{\mathrm{P}}=10$.

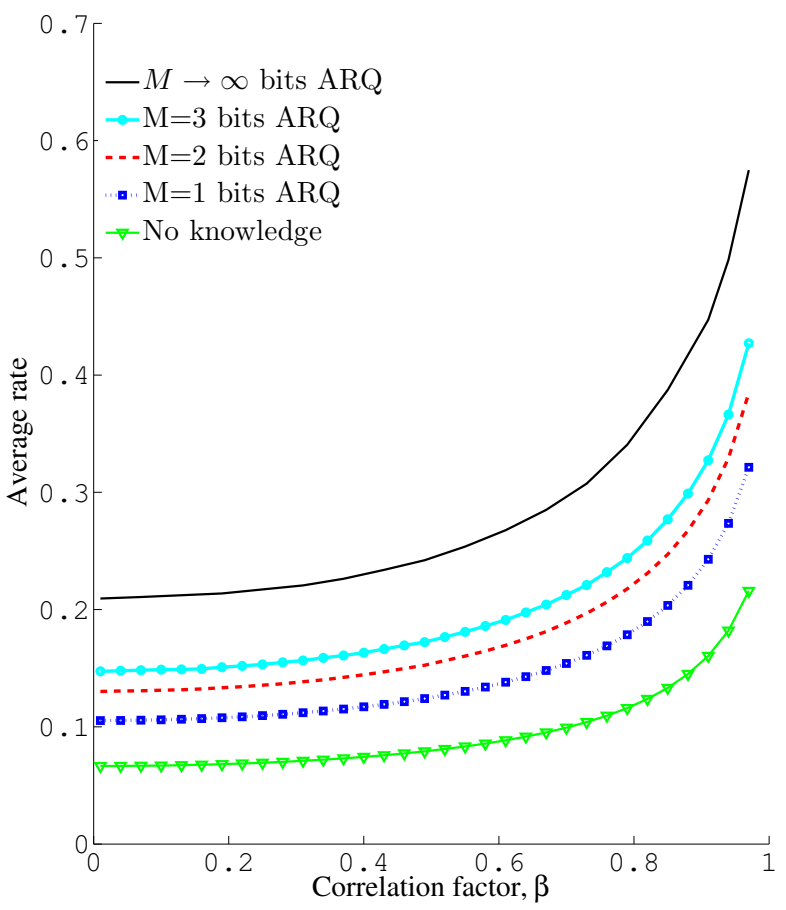

Figure 8. Average rate vs estimation factor $\beta$, imperfect CSI scenario, $P^{\mathrm{p}}=$ $2, I^{\mathrm{p}}=10, \pi=0.9$. 


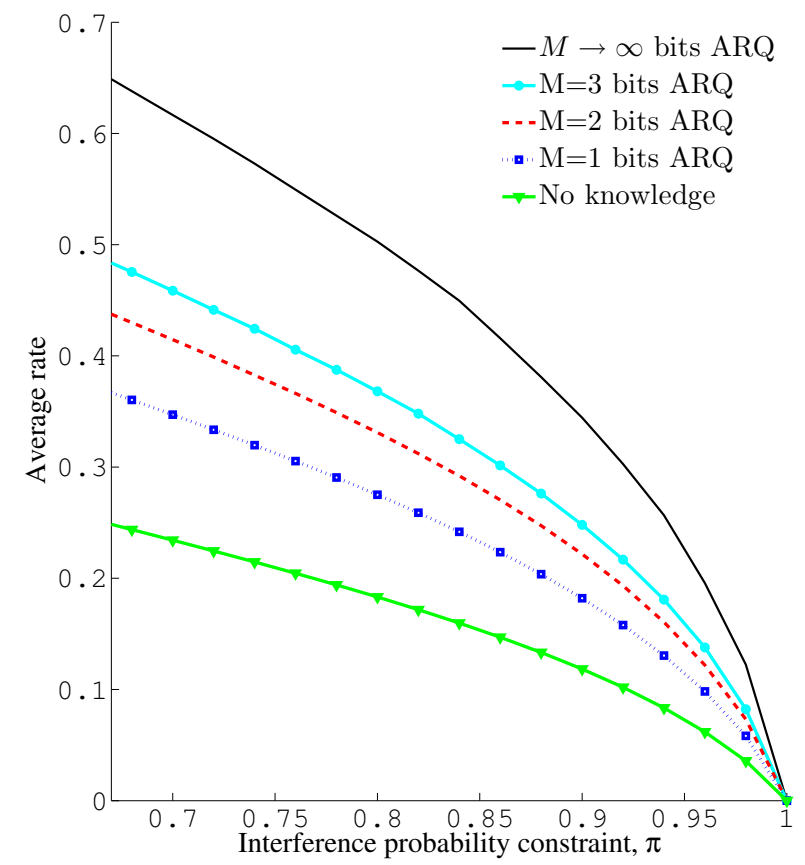

Figure 9. Average rate vs interference probability $\pi$, imperfect CSI scenario, $P^{\mathrm{p}}=2, I^{\mathrm{p}}=10, \beta=0.8$.

probability of the secondary user is obtained by

$$
\begin{aligned}
\operatorname{Pr}\left\{\text { outage }^{\mathrm{s}}\right. & =\operatorname{Pr}\left\{R_{M+1}^{\mathrm{s}}>\log \left(1+\frac{P g^{\mathrm{ss}}}{P^{\mathrm{p}} g^{\mathrm{ps}}+\sigma^{2}}\right)\right\} \\
& =\int_{0}^{\infty} f_{g^{\mathrm{ps}}}(y) \operatorname{Pr}\left\{g^{\mathrm{ss}}<\frac{\left(P^{\mathrm{p}} y+\sigma^{2}\right) \vartheta_{M+1}}{P}\right\} \mathrm{d} y \\
& =E_{g^{\mathrm{ps}}}\left\{F_{g^{\mathrm{ss}}}\left(\frac{\left(P^{\mathrm{p}} g^{\mathrm{ps}}+\sigma^{2}\right) \vartheta_{M+1}}{P}\right)\right\}
\end{aligned}
$$

Consequently, adding a new constraint $\operatorname{Pr}\left\{\right.$ outage $^{\mathrm{s}} \leq$ $\pi_{\text {outage }}$ to the previous peak power limited rate maximization problem, i.e., (11), results in a new optimization problem

$$
\begin{aligned}
& \bar{R}^{\mathrm{s}, \max }=\max _{R_{m}^{\mathrm{s}},} E_{m=1 \ldots M+1} E_{g^{\mathrm{ps}}}\left\{\sum _ { m = 1 } ^ { M + 1 } R _ { m } ^ { \mathrm { s } } \left(F_{g^{\mathrm{ss}}}\left(\frac{\left(P^{\mathrm{p}} g^{\mathrm{ps}}+\sigma^{2}\right) \vartheta_{m-1}}{P}\right)\right.\right. \\
& \left.\left.-F_{g^{\mathrm{ss}}}\left(\frac{\left(P^{\mathrm{p}} g^{\mathrm{ps}}+\sigma^{2}\right) \vartheta_{m}}{P}\right)\right)\right\}
\end{aligned}
$$$$
\text { subject to } E_{g^{\mathrm{ps}}}\left\{F_{g^{\mathrm{ss}}}\left(\frac{\left(P^{\mathrm{p}} g^{\mathrm{ps}}+\sigma^{2}\right) \vartheta_{M+1}}{P}\right)\right\} \leq \pi_{\text {outage }}
$$

that can be solved numerically. Note that for Rayleigh-fading channels (42) is simplified to

$$
\begin{aligned}
\operatorname{Pr}\{\text { outage }\}^{\mathrm{s}} & =\int_{0}^{\infty} \frac{1}{\lambda^{\mathrm{ps}}} e^{-\frac{y}{\lambda^{\mathrm{ps}}}}\left(1-e^{-\frac{\left(P^{\mathrm{p}} y+\sigma^{2}\right) \vartheta_{M+1}}{\lambda^{\mathrm{ss} P}}}\right) \mathrm{d} y \\
& =1-\frac{e^{-\frac{\sigma^{2} \vartheta_{M+1}}{\lambda^{\mathrm{ss} P}}}}{1+\frac{\lambda^{\mathrm{ps}} P^{\mathrm{p}} \vartheta_{M+1}}{\lambda^{\mathrm{ss}} P}}
\end{aligned}
$$

Considering limited received interference power condition, on the other hand, the outage probability of the secondary channel is formulated as

$$
\begin{aligned}
& \operatorname{Pr}\{\text { outage }\}^{\mathrm{s}}=\operatorname{Pr}\left\{R_{M+1}^{\mathrm{s}}>\log \left(1+\frac{I^{\mathrm{p}} g^{\mathrm{ss}}}{\tilde{g}^{\mathrm{sp}}\left(P^{\mathrm{p}} g^{\mathrm{ps}}+\sigma^{2}\right)}\right)\right\} \\
& =\int_{0}^{\infty} \int_{0}^{\infty} f_{\tilde{g}^{\mathrm{sp}}}(z) f_{g^{\mathrm{ps}}}(y) \operatorname{Pr}\left\{g^{\mathrm{ss}}<\frac{z\left(P^{\mathrm{p}} y+\sigma^{2}\right) \vartheta_{M+1}}{I^{\mathrm{p}}}\right\} \mathrm{d} z \mathrm{~d} y \\
& =E_{\tilde{g}^{\mathrm{sp}}, g^{\mathrm{ps}}}\left\{F_{g^{\mathrm{ss}}}\left(\frac{\tilde{g}^{\mathrm{sp}}\left(P^{\mathrm{p}} g^{\mathrm{ps}}+\sigma^{2}\right) \vartheta_{M+1}}{I^{\mathrm{p}}}\right)\right\}
\end{aligned}
$$

which for Rayleigh-fading channels leads to

$$
\begin{aligned}
& \operatorname{Pr}\{\text { outage }\}^{\mathrm{s}}=1- \\
& \frac{1}{\lambda^{\mathrm{ps}}} \frac{1}{\lambda^{\mathrm{sp}}} \int_{0}^{\infty} \int_{0}^{\infty} e^{-\left(\frac{y}{\lambda^{\mathrm{ps}}}+\frac{z}{\lambda^{\mathrm{sp}}}\right)} e^{-\frac{z}{\lambda^{\mathrm{ss}} I^{\mathrm{p}}}\left(P^{\mathrm{p}} y+\sigma^{2}\right) \vartheta_{M+1}} \mathrm{~d} z \mathrm{~d} y \\
& =1+\frac{\lambda^{\mathrm{ss}} I^{\mathrm{p}}}{\lambda^{\mathrm{ps}} \lambda^{\mathrm{sp}} P^{\mathrm{p}}} J\left(\vartheta_{M+1}\right) .
\end{aligned}
$$

Therefore, the optimal outage and interference power limited average rate of the channel is obtained by numerical solution of the problem

$$
\begin{aligned}
& \bar{R}^{\mathrm{s}, \max }= \max _{R_{m}^{\mathrm{s}}, \forall m} E_{\tilde{g}^{\mathrm{sp}}, g^{\mathrm{ps}}}\left\{\sum _ { m = 1 } ^ { M + 1 } R _ { m } ^ { \mathrm { s } } \left(F_{g^{\mathrm{ss}}}\left(\frac{\tilde{g}^{\mathrm{sp}}\left(P^{\mathrm{p}} g^{\mathrm{ps}}+\sigma^{2}\right) \vartheta_{m-1}}{I^{\mathrm{p}}}\right)\right.\right. \\
&\left.\left.-F_{g^{\mathrm{ss}}}\left(\frac{\tilde{g}^{\mathrm{sp}}\left(P^{\mathrm{p}} g^{\mathrm{ps}}+\sigma^{2}\right) \vartheta_{m}}{I^{\mathrm{p}}}\right)\right)\right\} \\
& \\
& \text { subject to } E_{\tilde{g}^{\mathrm{sp}}, g^{\mathrm{ps}}}\left\{F_{g^{\mathrm{ss}}}\left(\frac{\tilde{g}^{\mathrm{sp}}\left(P^{\mathrm{p}} g^{\mathrm{ps}}+\sigma^{2}\right) \vartheta_{M+1}}{I^{\mathrm{p}}}\right)\right\} \leq \pi_{\text {outage }}
\end{aligned}
$$

Finally, assuming a fixed SU transmission power, the outage probability of the primary user communicating at rate $R^{\mathrm{p}}$ is found as

$$
\begin{aligned}
\operatorname{Pr}\{\text { outage }\}^{\mathrm{p}} & =\operatorname{Pr}\left\{R^{\mathrm{p}}>\log \left(1+\frac{P^{\mathrm{p}} g^{\mathrm{pp}}}{P g^{\mathrm{sp}}+\sigma^{2}}\right)\right\} \\
& =E_{g^{\mathrm{sp}}}\left\{F_{g^{\mathrm{pp}}}\left(\frac{\left(P g^{\mathrm{sp}}+\sigma^{2}\right)\left(e^{R^{\mathrm{p}}}-1\right)}{P^{\mathrm{p}}}\right)\right\} .
\end{aligned}
$$

Therefore, for Rayleigh-fading channels, the SU transmission power satisfying a PU outage probability constraint $\pi_{\text {outage }}$, i.e, $\operatorname{Pr}\{\text { outage }\}^{\mathrm{p}} \leq \pi_{\text {outage }}$, is found as

$$
P=\max \left\{0, \frac{\lambda^{\mathrm{sp}} P^{\mathrm{p}}}{\lambda^{\mathrm{pp}}\left(e^{R^{\mathrm{p}}}-1\right)}\left(\frac{e^{-\lambda^{\mathrm{pp}}\left(\frac{e^{R^{\mathrm{p}}}-1}{P^{\mathrm{p}}}\right) \sigma^{2}}}{1-\pi_{\text {outage }}}-1\right)\right\}
$$

which will change the rate optimization problem correspondingly. Moreover, considering both SU transmission power and PU outage probability constraints, (49) can be used to determine the optimal SU transmission power, which will be the minimum of the powers obtained by these two constraints. Also, it is interesting to note that with proper parameter assignments the results obtained under limited SU transmission power condition, e.g., Fig. 2, can be represented as the ones under the PU outage probability constraint as well.

Implementing $M=1$ and 2 bits ARQ feedback and limited SU input power scenario, the average rates have been found for fixed primary user input power $P^{\mathrm{p}}=2$, as illustrated in Fig. 10. The SU outage probability constraint has been selected to be $\pi_{\text {outage }}=0.1$. Also, considering the same conditions, Fig. 11 demonstrates the SU-SU channel average rate under PU received interference limited scenario. Finally, setting $P^{\mathrm{p}}=$ 2 , the effect of the SU outage probability constraint on the average rate of the channel is further studied in Fig. 12 where the results are obtained for $M=0$ and 1 bit ARQ feedback and different power constraints. In this case, both SU input 


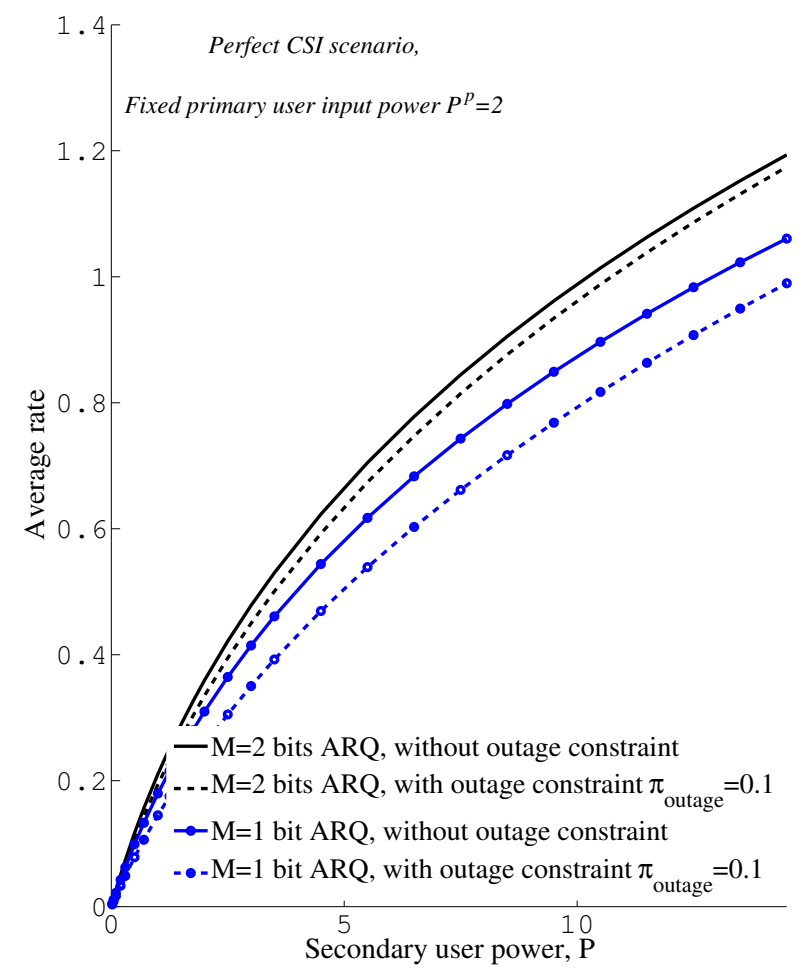

Figure 10. Outage limited average rate vs secondary user input power, perfect CSI scenario, $P^{\mathrm{P}}=2$.

power $P$ and PU received interference power $I^{\mathrm{p}}$ are selected to be limited by 10 . Also, considering $R^{\mathrm{p}}=0.3$ and $P^{\mathrm{p}}=2$, the figure represents the $\mathrm{SU}$ achievable rates in $\mathrm{PU}$ outage limited conditions.

\section{Discussions}

Theoretical and simulation results emphasize on a number of interesting points that can be listed as follows:

- The effect of HARQ feedback: While substantial performance improvement is achieved with even a single ARQ feedback round (Fig. 2 and 6), the presence of a primary user leads to drastic rate decrement for the secondary user (Fig. 3 and 7). This result is valid for both perfect and imperfect SU-PU CSI assumptions. Moreover, with the same total primary input power, increasing the number of primary users can drastically reduce the SU-SU channel average rate (Fig. 4). There is an interesting intuition behind this point; The SU average rate gains very much from the cases where the cross channels, and correspondingly the interferences, are weak. However, in a system with a number of PUs experiencing independent pdfs it is more likely that, at any time instant, some of the cross channels experience high gain realizations. Therefore, the SU average rate reduces since the received interference increases. Then, as seen in the figure, this effect becomes more critical as the number of ARQ rounds increases. On the other hand, increasing the number of primary users reduces the effect of hybrid ARQ feedback.

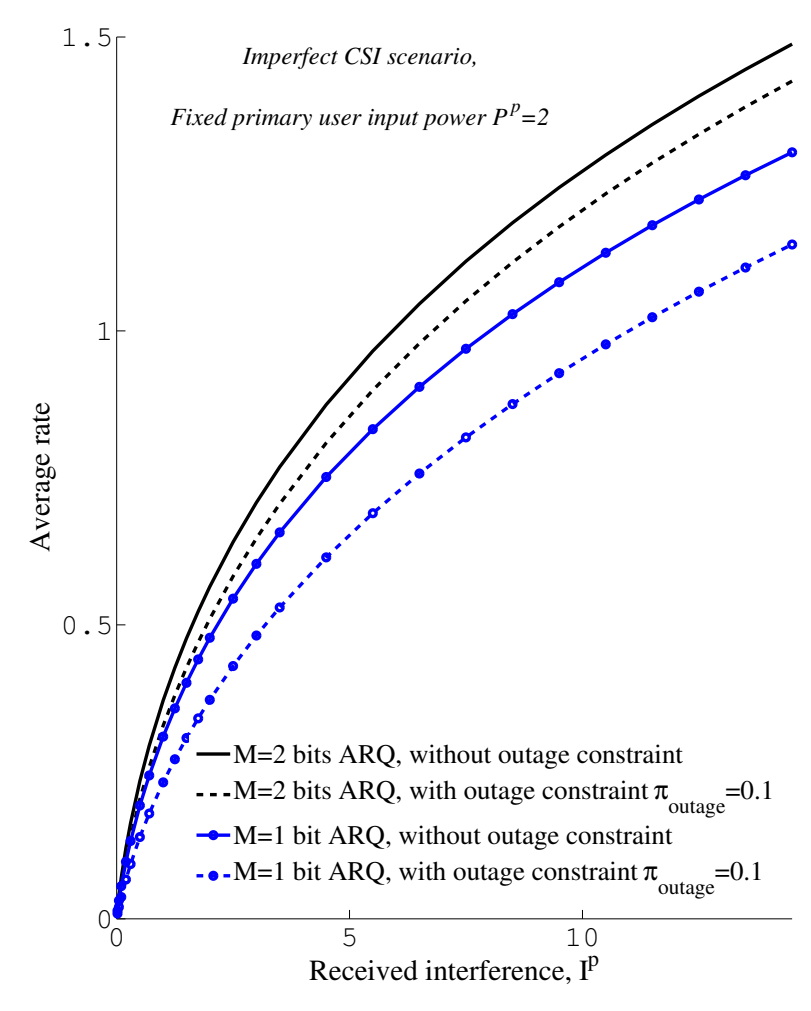

Figure 11. Outage limited average rate vs primary user received interference power, imperfect CSI scenario, $P^{\mathrm{P}}=2$.

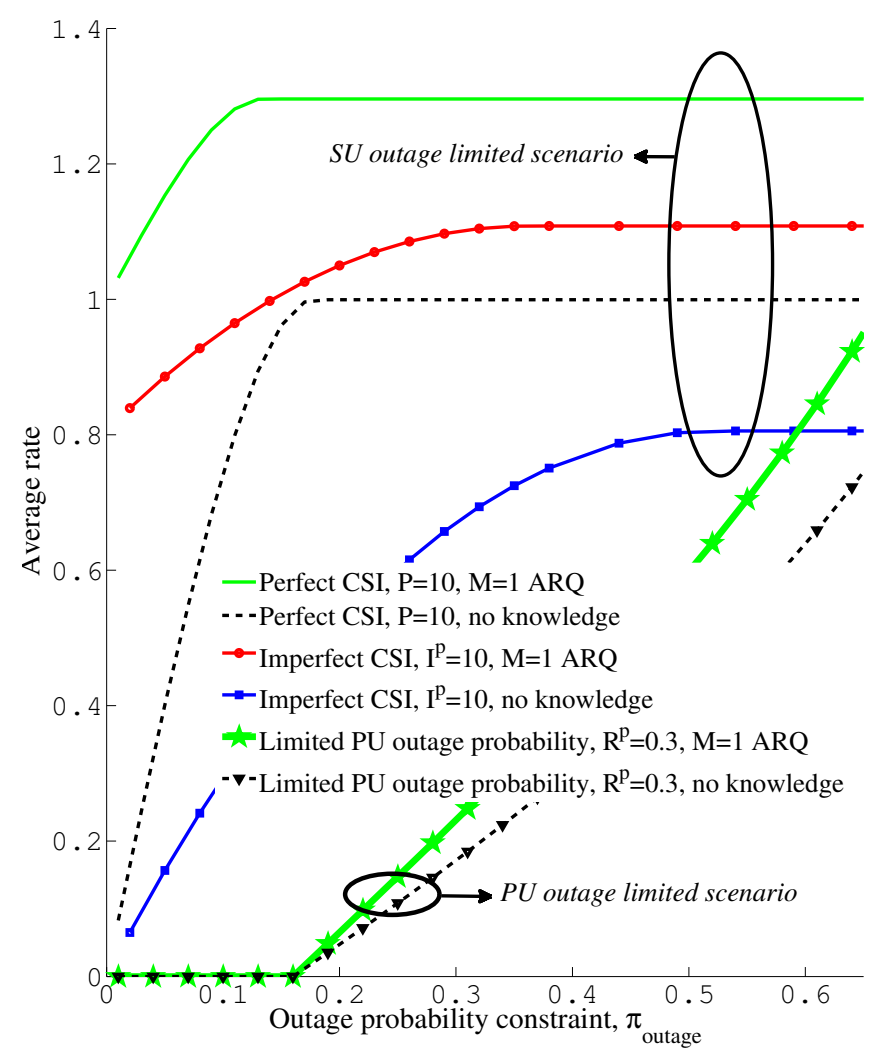

Figure 12. Average rate vs outage probability constraint. In all SU outage limited scenarios, the SU transmission power $P$ and the PU received interference power $I^{\mathrm{P}}$ are set to 10 . In PU outage limited conditions, we have $R^{\mathrm{p}}=0.3 . P^{\mathrm{p}}=2$. 
- Long-term vs short-term constraints: Considering limited SU input power (or equivalently, limited PU interference power with perfect SU-PU CSI scenario), optimal (longterm) power allocation increases the SU-SU channel average rate in low SINR conditions. However, this effect diminishes in high SINR regimes and the rates converge to the ones obtained with short-term power constraint (Fig. 5).

- The effect of imperfect CSI: The quality of the secondaryprimary channel state information can significantly affect the secondary channel average rate under interference limited conditions (Fig. 8). Moreover, having imperfect SU-PU CSI, the tolerance of the primary user, modeled by interference temperature probability $\pi$, plays a great role in the secondary channel average rate. That is, the more secure the interference temperature should be satisfied, the less average rate is achieved at the secondary channel, converging to zero (Fig. 9).

- The effect of the SU outage probability constraints: The presence of the SU outage probability constraint results in significant rate reduction under both perfect and imperfect CSI scenarios. However, increasing the ARQ retransmission rounds or the input power, the effect of the SU outage probability constraint diminishes and it is possible to reach (almost) the same performance as with no outage probability limitation (Fig. 10-12). Particularly, with infinite ARQ feedback bits the rates converge to the asymptotic channel performance bound with no SU outage probability constraint. Finally, although not seen in the figures, simulation results show that considering harder SU outage probability constraints, i.e., smaller

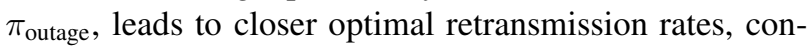
verging to origin.

- The effect of the PU outage probability constraints: While no data transmission is permitted under hard PU outage probability constraints, considerable transmission rates are achievable in the SU-SU channel under more relaxed PU outage limited conditions (Fig. 12).

\section{CONCLUSION}

This paper investigated the average rate of quasi-static spectrum sharing networks utilizing hybrid automatic repeat request feedback. The unlicensed secondary channel performance was studied in different situations. Under both peak and average input power constraints, the channel average rate was determined under primary user limited received interference power conditions when there is perfect or imperfect information about the interference available at the SU transmitter. Then, we proposed an approximate solution for power allocation between INR HARQ-based data retransmissions which can be applied in single-user networks as well. Finally, the last part of the paper focused on the effect of outage probability where, satisfying the previous constraints, the optimal average rates were found under outage-limited conditions. Simulation results show substantial performance improvement with even a single HARQ-based retransmission in all simulations. Finally, further studies about the system performance under both secondary user transmission power and primary user received interference power constraints are left for the future.

\section{REFERENCES}

[1] F. Berggren, O. Queseth, J. Zander, B. Asp, C. Jönsson, N. Z. Kviselius, B. Thorngren, U. Landmark, and J. Wessel, "Dynamic spectrum access, phase 1: scenarios and research challenges," Sept. 2004, available: http://www.queseth.se/olav/pubs/DSAReportPhase1.pdf.

[2] W. D. Horne, "Adaptive spectrum access: using the full spectrum space," 2003, available: http://intel.si.umich.edu/tprc/papers/2003/225/ Adaptive_Spectrum_Horne.pdf.

[3] R. H. Etkin, D. N. C. Tse, and H. Wang, "Gaussian interference channel capacity to within one bit," IEEE Transactions on Information Theory, vol. 54, no. 12, pp. 5534-5562, Dec. 2008.

[4] X. Shang, G. Kramer, and B. Chen, "A new outer bound and the noisyinterference sum-rate capacity for Gaussian interference channels," IEEE Transactions on Information Theory, vol. 55, no. 2, pp. 689-699, Feb. 2009.

[5] D. Tuninetti, "An outer bound region for interference channels with generalized feedback," in Information Theory and Applications Workshop, Jan. 2010, pp. 1-5.

[6] R. Narasimhan, "Hybrid-ARQ interference channels with receiver cooperation," IEEE International Conference on Communication, May 2010, to appear.

[7] - "Delay-limited throughput of cooperative multiple access channels with hybrid-ARQ," in IEEE International Symposium on Information Theory, July 2008, pp. 409-413.

[8] S.-Y. Jeon and D.-H. Cho, "An ARQ mechanism considering resource and traffic priorities in cognitive radio systems," IEEE Communications Letters, vol. 13, no. 7, pp. 504-506, July 2009.

[9] A. Jovicic and P. Viswanath, "Cognitive radio: An information-theoretic perspective," IEEE Transactions on Information Theory, vol. 55, no. 9, pp. 3945-3958, Sept. 2009.

[10] N. Devroye, P. Mitran, and V. Tarokh, "Achievable rates in cognitive radio channels," IEEE Transactions on Information Theory, vol. 52, no. 5, pp. 1813-1827, May 2006.

[11] - "Limits on communications in a cognitive radio channel," IEEE Communications Magazine, vol. 44, no. 6, pp. 44-49, June 2006.

[12] N. Devroye, M. Vu, and V. Tarokh, "Cognitive radio networks," IEEE Signal Processing Magazine, vol. 25, no. 6, pp. 12-23, Nov. 2008.

[13] S. M. Mishra, A. Sahai, and R. W. Brodersen, "Cooperative sensing among cognitive radios," in IEEE International Conference on Communications, vol. 4, June 2006, pp. 1658-1663.

[14] S. A. Jafar and S. Srinivasa, "Capacity limits of cognitive radio with distributed and dynamic spectral activity," IEEE Journal on Selected Areas in Communications, vol. 25, no. 3, pp. 529-537, April 2007.

[15] M. Gastpar, "On capacity under receive and spatial spectrum-sharing constraints," IEEE Transactions on Information Theory, vol. 53, no. 2, pp. 471-487, Feb. 2007.

[16] Y. Xing, C. N. Mathur, M. A. Haleem, R. Chandramouli, and K. P. Subbalakshmi, "Dynamic spectrum access with QoS and interference temperature constraints," IEEE Transactions on Mobile Computing, vol. 6, no. 4, pp. 423-433, April 2007.

[17] X. Kang, Y.-C. Liang, A. Nallanathan, H. K. Garg, and R. Zhang, "Optimal power allocation for fading channels in cognitive radio networks: Ergodic capacity and outage capacity," IEEE Transactions on Wireless Communications, vol. 8, no. 2, pp. 940-950, Feb. 2009.

[18] A. Ghasemi and E. S. Sousa, "Fundamental limits of spectrum-sharing in fading environments," IEEE Transactions on Wireless Communications, vol. 6, no. 2, pp. 649-658, Feb. 2007.

[19] C.-X. Wang, X. Hong, H.-H. Chen, and J. Thompson, "On capacity of cognitive radio networks with average interference power constraints," IEEE Transactions on Wireless Communications, vol. 8, no. 4, pp. 16201625, April 2009.

[20] J. Ji, W. Chen, H. Wan, and Y. Liu, "Capacity analysis of multicast network in spectrum sharing systems," IEEE International Conference on Communication, May 2010, to appear.

[21] L. Musavian and S. Aissa, "Capacity and power allocation for spectrumsharing communications in fading channels," IEEE Transactions on Wireless Communications, vol. 8, no. 1, pp. 148-156, Jan. 2009.

[22] _ - "Fundamental capacity limits of cognitive radio in fading environments with imperfect channel information," IEEE Transactions on Communications, vol. 57, no. 11, pp. 3472-3480, Nov. 2009. 
[23] H. A. Suraweera, P. J. Smith, and M. Shafi, "Capacity limits and performance analysis of cognitive radio with imperfect channel knowledge," IEEE Transactions on Vehicular Technology, vol. 59, no. 4, pp. 18111822, May 2010.

[24] L. Musavian and S. Aissa, "Outage-constrained capacity of spectrumsharing channels in fading environments," IET Communications, vol. 2, no. 6, pp. 724-732, July 2008.

[25] "Technical specification group radio access network, physical layer procedures," 3rd Generation Partnership Project, Tech. Spec. 3GPP ts 25.214 V3.10.0, 2002-2003.

[26] "Physical and medium access control layers for combined fixed and mobile operation in licensed bands," IEEE Std. Std 802.16e-2005, 2005.

[27] B. Makki, L. Beygi, and T. Eriksson, "Channel capacity bounds in the presence of quantized channel state information," in Eura. J. on Wireless Commun. and Net., vol. 2010, Dec. 2010, article ID 495014, doi: $10.1155 / 2010 / 495014$.

[28] B. Makki and T. Eriksson, "Data transmission in the presence of noisy channel state feedback and outage probability constraint," International Symposium on Information Theory and Applications, pp. 458-463, Oct. 2010.

[29] S. Ekbatani, F. Etemadi, and H. Jafarkhani, "Throughput maximization over slowly fading channels using quantized and erroneous feedback," IEEE Transaction on Communications, vol. 57, no. 9, pp. 2528-2533, Sept. 2009.

[30] B. Makki and T. Eriksson, "On the average rate of quasi-static fading channels with ARQ and CSI feedback," IEEE Communication Letters, vol. 14 , no. 9, pp. 806-808, June 2010.

[31] C. Shen, T. Liu, and M. P. Fitz, "On the average rate performance of hybrid-ARQ in quasi-static fading channels," IEEE Transaction on Communications, vol. 57, no. 11, pp. 3339-3352, Nov. 2009.

[32] D. Tuninetti, "Transmitter channel state information and repetition protocols in block fading channels," in IEEE Information Theory Workshop, Sept. 2007, pp. 505-510.

[33] J. Perret and D. Tuninetti, "Repetition protocols for block fading channels that combine transmission requests and state information," in IEEE International Conference on Communications, May 2008, pp. 1297-1301.

[34] H. E. Gamal, G. Caire, and M. O. Damen, "The diversity-multiplexingdelay tradeoff in MIMO ARQ channels," in International Symposium on Information Theory, Sept. 2005, pp. 1823-1827.

[35] P. Wu and N. Jindal, "Performance of hybrid-ARQ in block-fading channels: A fixed outage probability analysis," IEEE Transaction on Communications, vol. 58, no. 4, pp. 1129-1141, April 2010.

[36] W. Su, S. Lee, D. A. Pados, and J. D. Matyjas, "The optimal transmission power per round for hybrid-ARQ rayleigh fading links," in IEEE International Conference on Communications, May 2010, to appear.

[37] M. Vu, "Exploiting transmit channel side information in MIMO wireless systems," Ph.D. dissertation, Stanford University, Stanford, CA, 2006.

[38] F. E. Lapiccirella, Z. Ding, and X. Liu, "Cognitive spectrum access control based on intrinsic primary ARQ information," in IEEE International Conference on Communication, May 2010, to appear.

[39] W. C. Ao and K. C. Chen, "End-to-end HARQ in cognitive radio networks," in IEEE Wireless Communications and Networking Conference, 2010, pp. 1-6.

[40] K. Eswaran, M. Gastpar, and K. Ramchandran, "Bits through ARQs: Spectrum sharing with a primary packet system," in IEEE International Symposium on Information Theory, June 2007, pp. 2171-2175.

[41] R. A. Tannious and A. Nosratinia, "Cognitive radio protocols based on exploiting hybrid ARQ retransmissions," IEEE Transactions on Wireless Communications, vol. 9, no. 9, pp. 2833-2841, 2010.

[42] B. Zhao and M. C. Valenti, "Practical relay networks: a generalization of hybrid-ARQ," IEEE Journal on Selected Areas in Communications, vol. 23, no. 1, pp. 7-18, Jan. 2005.

[43] L. Badia, M. Rossi, and M. Zorzi, "SR ARQ packet delay statistics on markov channels in the presence of variable arrival rate," IEEE Transaction on Wireless Communications, vol. 5, no. 7, pp. 1639-1644, July 2006.

[44] R. Narasimhan, "Individual outage rate regions for fading multiple access channels," in IEEE International Symposium on Information Theory, June 2007, pp. 1571-1575.

[45] T. M. Cover and J. A. Thomas, Elements of Information Theory. New York: Wiley Interscience, 1992.

[46] S. Sesia, G. Caire, and G. Vivier, "Incremental redundancy hybrid ARQ schemes based on low-density parity-check codes," IEEE Transactions on Communications, vol. 52, no. 8, pp. 1311-1321, Aug. 2004.
[47] J. Kim, W. Hur, A. Ramamoorthy, and S. W. McLaughlin, "Design of rate-compatible irregular LDPC codes for incremental redundancy hybrid ARQ systems," in IEEE International Symposium on Information Theory, July 2006, pp. 1139-1143.

[48] N. Varnica, E. Soljanin, and P. Whiting, "LDPC code ensembles for incremental redundancy hybrid ARQ," in International Symposium on Information Theory, Sept. 2005, pp. 995-999.

[49] "Trellisware technologies, white papers on flexicodes and F-LDPC," available: www.trellisware.com.

[50] A. J. Goldsmith and P. P. Varaiya, "Capacity of fading channels with channel side information," IEEE Transaction on Information Theory, vol. 43, no. 6, pp. 1986-1992, Nov. 1997.

[51] J. Peha, "Approaches to spectrum sharing," IEEE Communications Magazine, vol. 43, no. 2, pp. 10-12, Feb. 2005.

[52] K. S. Ahn and R. W. Heath, "Performance analysis of maximum ratio combining with imperfect channel estimation in the presence of cochannel interferences," IEEE Transactions on Wireless Communications, vol. 8, no. 3, pp. 1080-1085, March 2009.

[53] Q. Sun, D. C. Cox, H. C. Huang, and A. Lozano, "Estimation of continuous flat fading MIMO channels," IEEE Transactions on Wireless Communications, vol. 1, no. 4, pp. 549-553, Oct. 2002.

[54] T. L. Marzetta, "BLAST training: Estimating channel characteristics for high capacity space-time wireless," in 37th Annual Allerton Conference on Communication, Control and Computing, vol. 1, no. 4, Sept. 1999, pp. $958-966$.

[55] S. M. Almalfouh and G. L. Stuber, "Interference-aware power allocation in cognitive radio networks with imperfect spectrum sensing," IEEE International Conference on Communication, May 2010, to appear.

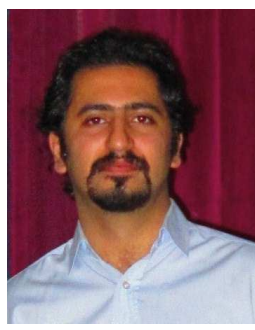

Behrooz Makki was born in Tehran, Iran. He received the B.Sc. degree in Electrical Engineering from Sharif University of Technology, Tehran, Iran, and the M.Sc. degree in Bioelectric Engineering from Amirkabir University of Technology, Tehran, Iran, in 2005 and 2008, respectively. Since 2009, he is working for his $\mathrm{PhD}$ degree in Communication Engineering at Chalmers University of Technology, Gothenburg, Sweden. His current research interests include partial channel state information (CSI) feedback, hybrid automatic repeat request, correlated fading channels and spectrum sharing networks.

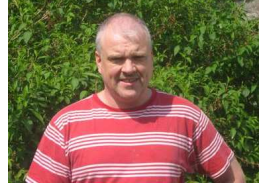

Thomas Eriksson received the Ph.D. degree in Information Theory in 1996, from Chalmers University of Technology, Gothenburg, Sweden. From 1990 to 1996, he was at Chalmers. In 1997 and 1998, he was at ATT Labs - Research in Murray Hill, NJ, USA, and in 1998 and 1999 he was at Ericsson Radio Systems AB, Kista, Sweden. Since 1999, he has been at Chalmers University, where he is currently a professor in communication systems. Further, he was a guest professor at Yonsei University, Seoul, S. Korea, in 2003-2004. His research interests include communication, data compression, and modeling and compensation of nonideal hardware components (e.g. amplifiers and oscillators in communication transmitters and receivers). 